aao3526 Bradley preedit

\title{
Title:
}

\section{Evolution of flower color pattern through selection on regulatory small RNAs}

\begin{abstract}
Authors:
Desmond Bradley ${ }^{1}$, Ping $\mathrm{Xu}^{2}$, Irina-Ioana Mohorianu ${ }^{3}$, Annabel Whibley ${ }^{1}$, David Field ${ }^{4,5}$, Hugo Tavares ${ }^{1,9}$, Matthew Couchman ${ }^{1}$, Lucy Copsey ${ }^{1}$, Rosemary Carpenter ${ }^{1}$, Miaomiao $\mathrm{Li}^{6,7}$, Qun $\mathrm{Li}^{6}$, Yongbiao Xue ${ }^{6,7,8}$, Tamas Dalmay ${ }^{2 *}$, Enrico Coen ${ }^{1 *}$
\end{abstract}

Affiliations:

${ }^{1}$ Department of Cell and Developmental Biology, John Innes Center, Colney Lane, Norwich NR4 7UH, UK.

${ }^{2}$ School of Biological Sciences, University of East Anglia, Norwich, NR4 7TJ. 
${ }^{3}$ School of Biological Sciences and School of Computing Sciences, University of East Anglia, Norwich, NR4 7TJ.

${ }^{4}$ Department of Botany and Biodiversity Research, University of Vienna, Faculty of Life Sciences, Rennweg 14, A-1030 Vienna, Austria.

${ }^{5}$ IST Austria, Klosterneuburg, Austria.

${ }^{6}$ State Key Laboratory of Molecular Developmental Biology, Institute of Genetics and Developmental Biology, Chinese Academy of Sciences and National Center for Plant Gene Research, Beijing 100101, China.

${ }^{7}$ University of Chinese Academy of Sciences, Beijing 100190, China.

${ }^{8}$ Beijing Institute of Genomics, Chinese Academy of Sciences, Beijing 100101, China.

${ }^{9}$ Current address: Sainsbury Laboratory, University of Cambridge, Cambridge CB2 1LR, UK.

*Corresponding authors

Abstract: 
Small RNAs regulate genes in plants and animals. Here we show that populationwide differences in color patterns in snapdragon flowers are caused by an inverted duplication that generates small RNAs. The complexity and size of the transcripts indicate the duplication represents an intermediate on the pathway to microRNA evolution. The small RNAs repress a pigment biosynthesis gene, creating a yellow highlight at the site of pollinator entry. The inverted duplication exhibits steep clines in allele frequency in a natural hybrid zone, showing that the allele is under selection. Thus, regulatory interactions of evolutionarily recent small RNAs can be acted upon by selection and contribute to the evolution of phenotypic diversity.

One Sentence Summary:

Selection acting on an inverted duplication that generates small RNAs leads to evolution of regulatory interactions and phenotypic change.

\section{LENGTH}

\section{Main Text:}

A convenient system for studying selection in natural populations is afforded by hybrid zones, where closely-related species or populations come into contact (1). Such a hybrid zone has been described for two subspecies of Antirrhinum majus (snapdragon), that 
differ in flower color (2), a trait involved in pollinator attraction (3-7). Both subspecies are pollinated by bees but have alternate patterns for guiding flower entry:

A.m.pseudomajus flowers are magenta, with a patch of yellow highlighting the bee entry point (Fig. 1A), whereas A.m.striatum flowers are yellow with magenta veins at the entry point (Fig. 1B). The magenta and yellow flower color intensities show sharp clines at a hybrid zone (2) where the subspecies come into contact. Production of magenta is regulated by ROSEA (ROS) and ELUTA (EL) (8-10). ROS encodes a MYB-like transcription factor that promotes anthocyanin biosynthetic gene expression in A.m.pseudomajus and exhibits a steep cline in allele frequencies at the hybrid zone $(2,9)$. Distribution of yellow pigment is regulated by $S U L F$ (Fig. 1B, C), which represses production of the yellow flavonoid aurone in A.m.pseudomajus (Fig. 1D) (2, 9, 10). Here we study the molecular nature of SULF.

To isolate SULF we first mapped it to an interval of $\sim 3 \mathrm{Mb}$ on chromosome 4 by sequencing pools of sulf and SULF phenotypes from a segregating population (fig. S1). In parallel, we carried out a transposon mutagenesis experiment in A. majus (SULF) and isolated a mutant, sulf-660, that was both somatically and genetically unstable (fig. S2A and Methods). Comparing the genome sequence of sulf-660 and its revertants revealed a single insertion site, within the mapped region of SULF, specific to sulf-660. Three independent revertants had different excision footprints at this site, confirming that the transposon was responsible for the sulf phenotype (fig. S2B). 
BLAST searches of the sequence flanking the transposon insertion site revealed regions of $74-88 \%$ nucleotide sequence identity to A.majus chalcone 4'-O-gluosyltransferase (Am4'CGT), which encodes an enzyme involved in synthesis of the yellow pigment aurone (Fig. 2A and table S1) (11). The regions of Am4'CGT homology were organized as an inverted duplication in the A. majus SULF genome. Both the left and right arms of the duplication carried deletions relative to intact $A m 4$ 'CGT, suggesting they had independently degenerated from a more complete precursor. A contiguous region of inverted homology between the left and right arms spanned a 590 bp region (red arrows, Fig. 2A), separated by a $\sim 600$ bp spacer region, which contained the transposon insertion site of sulf-660. Phylogenetic analysis indicated that the SULF inverted repeats were likely generated from Am4'CGT recently in the evolution of the Antirrhinum lineage (Fig. 2B and fig. S3).

To determine whether the inverted duplication at SULF might be under selection, we compared A.m.pseudomajus and A.m.striatum populations sampled either side of a hybrid zone. PCR using oligos flanking the inverted repeats gave bands in the range 1.5-2.5 kb for all individuals from the A.m.pseudomajus ( $\mathrm{n}=96$ ) but not the A.m.striatum populations ( $\mathrm{n}=95)$, suggesting that the inverted duplication was present at higher frequency in A.m.pseudomajus (fig. S4). Sequencing pools of $\sim 50$ individuals from each population revealed reduced depth of sequence for A.m.striatum compared to A.m.pseudomajus over a $\sim 145 \mathrm{~kb}$ region around SULF, suggesting that A.m.striatum carried deletions relative to A.m.pseudomajus in this chromosome region (Fig. 2C). 
This conclusion was supported by PCR amplification assays using a range of oligos. Deletion alleles were also observed in resequenced individuals, including a $1.3 \mathrm{~kb}$ deletion that removed the left arm of the inverted repeat and part of the spacer sequence in A.m. striatum pools. Thus, the inverted duplication present in SULF of A.m.pseudomajus is absent or at low frequency in A.m.striatum populations, further demonstrating the requirement for the inverted duplication for SULF function.

SNPs in a $\sim 300 \mathrm{~kb}$ interval containing SULF, showed steep clines in allele frequency (Fig. 2D and fig. S5), centered at the same geographic location as clines for $R O S$ and flower color (2). SNPs sampled from other positions along chromosome 4 either showed no clines, or clines centered at different geographic locations (Fig. 2D and fig. S5). The significance of the clines at $S U L F$ was confirmed by comparing DNA sequences from pools of individuals sampled from a transect covering 20km either side of the hybrid zone. Of the $\sim 7 \times 10^{5}$ polymorphic SNPs on the SULF chromosome, 99\% showed no allele frequency differences across the transect, and of those that did, more than $99 \%$ did not give steep clines aligned with ROS. Thus, there is likely to be strong selection acting on SULF.

The coincidence of the SULF and ROS clines suggests that these loci interact. In A.m. pseudomajus, where ROS confers magenta color, SULF could be favored because it restricts yellow to create a contrasting highlight at the bee entry point (Fig. 1A). In A.m.striatum, where ros confers reduced magenta intensity for much of the flower, sulf could be favored because it confers both a striking yellow color and a contrasting 
background to the magenta veins (Fig. 1B). Thus, selection acting on different allele combinations at $S U L F$ and $R O S$ allows alternate floral guides to be maintained either side of a hybrid zone. The situation is comparable to selection acting on loci controlling yellow and red coloration of mimetic patterns in Heliconius butterflies $(12,13)$.

Given the structure of the inverted duplication at SULF and its homology to Am4'CGT, we hypothesized that SULF represses Am4'CGT, and thus restricts yellow flower color, via regulatory small RNAs. To determine whether SULF generated small RNAs, small RNA libraries were prepared from petals of A.majus SULF and sulf-660. The biggest differences in small RNA abundance mapped to the SULF inverted repeats and corresponded to predominantly 21-mers (Fig. 3A, B). RNA blots probed with SULF confirmed small RNAs from the inverted repeat were present in SULF and absent in sulf genotypes, including A.m.striatum (Fig. 3C and fig. S6). The small RNAs likely derive from processed transcripts predicted to generate long foldback hairpin RNAs (fig. S7).

If the small RNAs generated by SULF restrict yellow pigmentation by targeting Am4'CGT, then SULF and Am4'CGT should exhibit complementary expression patterns. Analysis of RNA extracted from yellow and non-yellow regions of the petals of A.majus showed that SULF was preferentially expressed in the non-yellow region, whereas Am4'CGT was mainly expressed in the yellow region (Fig. 3D). The spatial restriction of Am4'CGT was confirmed by RNA in situ hybridization (Fig. 3E).

Overall expression of Am4'CGT was lower in petals of SULF compared to sulf-660 (Fig. 3F). 5' RACE on SULF genotypes revealed products for Am4'CGT terminating at a 
range of positions, suggesting cleavage at multiple sites (fig. S8). No cleavage products were found in sulf. The lack of a single cleavage site in SULF genotypes is consistent with the SULF inverted duplication generating multiple small RNAs targeting Am4'CGT (Fig. 3B). To determine whether SULF alleles from the subspecies also varied in their ability to repress $A m 4^{\prime} C G T$, we introgressed SULF from A.m.pseudomajus $\left(S U L F^{p}\right)$ or A.m.striatum (sulf $f^{s}$ ) into an A.majus background with the same Am4'CGT target allele. Am4'CGT expression was reduced in both dorsal and ventral petals of $S U L F^{P}$ compared to sulf (Fig. 3F). Thus, SULF acts by repressing transcript levels of the target Am4'CGT gene in A.m.pseudomajus but not in A.m.striatum.

If selection on inverted duplications is a common mechanism for establishing regulatory interactions, we might expect the genome to contain a large number of inverted duplications similar to SULF. Scanning the A.majus genome for inverted duplications with a similar adjusted folding energy to SULF revealed many such regions, some of which generated small RNAs (Fig. 4A). However, most of these small RNAs were $>21 \mathrm{nt}$ long, unlike those generated by SULF (circled, Fig. 4B), which were 21nt. Moreover, the small RNA population generated by SULF was of relatively low complexity because of the high abundance of a subset of small RNAs. Based on size and complexity, the profile of small RNAs generated by SULF was similar to that of conserved microRNAs (orange spots, Fig. 4B). Given the SULF hairpin is about five times longer than a typical conserved microRNA hairpin, these findings suggest that $S U L F$ generates a functioning long regulatory hairpin RNA. 
If only a subset of small RNAs generated by SULF are required to inhibit target gene activity, selection would not be able to maintain homology with the target gene Am4'CGT over the extended length observed (590bp). This argument implies that SULF is of recent evolutionary origin, consistent with the phylogenetic analysis (Fig. 2B). With respect to its young age, SULF is similar to other inverted duplications with extended similarity to protein coding regions that encode small RNAs (14-17). Over evolutionary time, functional inverted duplications such as SULF might be lost, maintained, or become shorter microRNA hairpins $(14,15,18-21)$. The deletions observed in both the left and right arms of the inverted repeat at $S U L F$, relative to Am4'CGT (Fig. 2A), suggest the process of size reduction may have already occurred to some extent. Among the many documented cases of loci contributing to natural variation (22), several examples of small regulatory RNAs have been described (23-26). However, these examples involve changes in expression pattern of pre-existing micro-RNAs or creation of new target sites, rather than de novo generation of a small regulatory RNA, as observed with SULF. The unusual nature of SULF may be a matter of chance or may reflect constraints on regulatory mechanisms (27). For example, the biosynthetic pathway to yellow aurone pigment synthesis has fewer steps and has a more limited taxonomic distribution than the magenta anthocyanin pigment synthesis pathway (11, 28). Variation in transcription factors, such as ROS, may therefore not be available specifically to modulate yellow patterning. Inverted duplications that generate regulatory RNAs may thus provide a flexible mechanism, complementing that based on transcription factor or cis-regulatory variation (22), for modulating or creating novel 
expression patterns upon which natural selection may act to generate evolutionary change.

\section{References and Notes}

1. N. H. Barton, G. M. Hewitt, Adaptation, speciation and hybrid zones. Nature 341, 497-503 (1989).

2. A. C. Whibley et al., Evolutionary paths underlying flower color variation in Antirrhinum. Science 313, 963-966 (2006).

3. R. Hopkins, M. D. Rausher, Identification of two genes causing reinforcement in the Texas wildflower Phlox drummondii. Nature 469, 411-414 (2011).

4. H. D. Bradshaw, D. W. Schemske, Allele substitution at a flower colour locus produces a pollinator shift in monkeyflowers. Nature 426, 176-178 (2003).

5. Y. W. Yuan, K. J. Byers, H. D. Bradshaw, Jr., The genetic control of flowerpollinator specificity. Curr Opin Plant Biol 16, 422-428 (2013).

6. H. Sheehan et al., MYB-FL controls gain and loss of floral UV absorbance, a key trait affecting pollinator preference and reproductive isolation. Nat Genet 48, 159166 (2016).

7. M. T. Clegg, M. L. Durbin, Tracing floral adaptations from ecology to molecules. Nat Rev Genet 4, 206-215 (2003).

8. J. Hackbarth, P. Michaelis, G. Scheller, Z. Indukt. Abstammungs-Vererbungslehre 80, 1 (1942).

9. K. Schwinn et al., A small family of MYB-regulatory genes controls floral pigmentation intensity and patterning in the genus Antirrhinum. Plant Cell 18, 831-851 (2006).

10. H. Stubbe, Genetik und Zytologie von Antirrhinum L. sect. Antirrhinum. (Veb Gustav Fischer Verlag, Jena, Germany, 1966).

11. E. Ono et al., Yellow flowers generated by expression of the aurone biosynthetic pathway. Proc Natl Acad Sci U S A 103, 11075-11080 (2006).

12. C. D. Jiggins, The Ecology and Evolution of Heliconius Butterflies. (Oxford Univeristy Press, 2017).

13. N. J. Nadeau, Genes controlling mimetic colour pattern variation in butterflies. Curr Opin Insect Sci 17, 24-31 (2016).

14. N. Fahlgren et al., MicroRNA gene evolution in Arabidopsis lyrata and Arabidopsis thaliana. Plant Cell 22, 1074-1089 (2010).

15. E. Allen et al., Evolution of microRNA genes by inverted duplication of target gene sequences in Arabidopsis thaliana. Nat Genet 36, 1282-1290 (2004). 
16. M. J. Axtell, Evolution of microRNAs and their targets: are all microRNAs biologically relevant? Biochim Biophys Acta 1779, 725-734 (2008).

17. O. Voinnet, Origin, biogenesis, and activity of plant microRNAs. Cell 136, 669687 (2009).

18. J. Cui, You, C., Chen, X., The evolution of microRNAs in plants. Current Opinions in Plant Biology 35, 61-67 (2017).

19. J. Piriyapongsa, Jordan, I.K., A Family of Human MicroRNA Genes from Minature Inverted-Repeat Transposable Elements. PLOS one 2, e203 (2007).

20. M. Nozawa, S. Miura, M. Nei, Origins and evolution of microRNA genes in plant species. Genome Biol Evol 4, 230-239 (2012).

21. F. Borges, R. A. Martienssen, The expanding world of small RNAs in plants. Nat Rev Mol Cell Biol 16, 727-741 (2015).

22. A. Martin, V. Orgogozo, The Loci of repeated evolution: a catalog of genetic hotspots of phenotypic variation. Evolution 67, 1235-1250 (2013).

23. S. Arif et al., Evolution of mir-92a underlies natural morphological variation in Drosophila melanogaster. Curr Biol 23, 523-528 (2013).

24. A. Clop et al., A mutation creating a potential illegitimate microRNA target site in the myostatin gene affects muscularity in sheep. Nat Genet 38, 813-818 (2006).

25. S. K. Nair et al., Cleistogamous flowering in barley arises from the suppression of microRNA-guided HvAP2 mRNA cleavage. Proc Natl Acad Sci U S A 107, 490495 (2010).

26. J. M. Debernardi, H. Lin, G. Chuck, J. D. Faris, J. Dubcovsky, microRNA172 plays a crucial role in wheat spike morphogenesis and grain threshability. Development 144, 1966-1975 (2017).

27. K. Chen, N. Rajewsky, The evolution of gene regulation by transcription factors and microRNAs. Nat Rev Genet 8, 93-103 (2007).

28. Y. Tanaka, N. Sasaki, A. Ohmiya, Biosynthesis of plant pigments: anthocyanins, betalains and carotenoids. Plant J 54, $733-749$ (2008).

29. I. Mohorianu et al., Profiling of short RNAs during fleshy fruit development reveals stage-specific sRNAome expression patterns. Plant J 67, 232-246 (2011).

30. R. Carpenter, Martin, C., Coen, E.S., Comparison of genetic behaviour of the transposable element Tam3 at two unlinked pigment loci in Antirrhinum majus. . Molecular and General Genetics 207, 82-89 (1987).

31. C. Martin, A. Prescott, S. Mackay, J. Bartlett, E. Vrijlandt, Control of anthocyanin biosynthesis in flowers of Antirrhinum majus. Plant J 1, 37-49 (1991).

32. K. M. Davies, Marshall, G.B., Bradley, J.M., Schwinn, K.E., Bloor, S.J., Winefield, C.S., Martin, C.R., Characterisation of aurone biosynthesis in Antirrhinum majus. Physiologia Plantarum 128, 593-603 (2006).

33. B. J. Harrison, Carpenter, R., Resurgence of genetic instability in Antirrhinum majus. Mutation Research 63, 47-66 (1979).

34. H. Sommer, Carpenter, R., Harrison, B.J., Saedler, H., The transposable element, Tam3, of Antirrhinum majus generates a novel type of sequence alteration upon excision. Molecular and General Genetics 199, 225-231 (1985).

35. R. Carpenter, E. S. Coen, Floral homeotic mutations produced by transposonmutagenesis in Antirrhinum majus. Genes Dev 4, 1483-1493 (1990). 
36. E. S. Coen, R. Carpenter, C. Martin, Transposable elements generate novel spatial patterns of gene expression in Antirrhinum majus. Cell 47, 285-296 (1986).

37. J. M. Szymura, N. H. Barton, Genetic Analysis of a Hybrid Zone between the Fire-Bellied Toads, Bombina Bombina and B. Variegata, near Cracow in Southern Poland. Evolution 40, 1141-1159 (1986).

38. P. Xu, Billmeier, M., Mohorianu, I., Green, D., Fraser, W.D., Dalmay, T., An improved protocol for small RNA library construction using High Definition adapters. Methods Next Generation Seq 2, 1-10 (2015).

39. S. Lopez-Gomollon, Detecting sRNAs by Northern blotting. Methods Mol Biol 732, 25-38 (2011).

40. C. Llave, Z. Xie, K. D. Kasschau, J. C. Carrington, Cleavage of Scarecrow-like mRNA targets directed by a class of Arabidopsis miRNA. Science 297, 20532056 (2002).

41. A. B. Rebocho, P. Southam, J. R. Kennaway, J. A. Bangham, E. Coen, Generation of shape complexity through tissue conflict resolution. Elife 6, (2017).

42. F. Piron Prunier, M. Chouteau, A. Whibley, M. Joron, V. Llaurens, Selection of Valid Reference Genes for Reverse Transcription Quantitative PCR Analysis in Heliconius numata (Lepidoptera: Nymphalidae). J Insect Sci 16, (2016).

43. E. Allen, Z. Xie, A. M. Gustafson, J. C. Carrington, microRNA-directed phasing during trans-acting siRNA biogenesis in plants. Cell 121, 207-221 (2005).

44. K. Sorefan et al., Reducing ligation bias of small RNAs in libraries for next generation sequencing. Silence 3, 4 (2012).

45. Mohorianu, II et al., Genomic responses to socio-sexual environment in male Drosophila melanogaster exposed to conspecific rivals. RNA, (2017).

46. M. Beckers et al., Comprehensive processing of high-throughput small RNA sequencing data including quality checking, normalization, and differential expression analysis using the UEA sRNA Workbench. RNA 23, 823-835 (2017).

47. K. Prufer et al., PatMaN: rapid alignment of short sequences to large databases. Bioinformatics 24, 1530-1531 (2008).

48. N. A. Fonseca, J. Rung, A. Brazma, J. C. Marioni, Tools for mapping highthroughput sequencing data. Bioinformatics 28, 3169-3177 (2012).

49. A. Kozomara, S. Griffiths-Jones, miRBase: annotating high confidence microRNAs using deep sequencing data. Nucleic Acids Res 42, D68-73 (2014).

50. A. Mortazavi, B. A. Williams, K. McCue, L. Schaeffer, B. Wold, Mapping and quantifying mammalian transcriptomes by RNA-Seq. Nat Methods 5, 621-628 (2008).

51. K. P. McCormick, M. R. Willmann, B. C. Meyers, Experimental design, preprocessing, normalization and differential expression analysis of small RNA sequencing experiments. Silence 2, 2 (2011).

52. M. A. Dillies et al., A comprehensive evaluation of normalization methods for Illumina high-throughput RNA sequencing data analysis. Brief Bioinform 14, 671-683 (2013).

53. I. Mohorianu, M. B. Stocks, J. Wood, T. Dalmay, V. Moulton, CoLIde: a bioinformatics tool for CO-expression-based small RNA Loci Identification using high-throughput sequencing data. RNA Biol 10, 1221-1230 (2013).

54. R. Lorenz et al., ViennaRNA Package 2.0. Algorithms Mol Biol 6, 26 (2011). 
55. P. Rice, I. Longden, A. Bleasby, EMBOSS: the European Molecular Biology Open Software Suite. Trends Genet 16, 276-277 (2000).

56. C. Ye, G. Ji, L. Li, C. Liang, detectIR: a novel program for detecting perfect and imperfect inverted repeats using complex numbers and vector calculation. PLoS One 9, e113349 (2014).

57. Y. Wang, J. M. Huang, Lirex: A Package for Identification of Long Inverted Repeats in Genomes. Genomics Proteomics Bioinformatics 15, 141-146 (2017).

58. M. B. Stocks et al., The UEA sRNA workbench: a suite of tools for analysing and visualizing next generation sequencing microRNA and small RNA datasets. Bioinformatics 28, 2059-2061 (2012).

\section{Acknowledgments}

The sRNA-seq data presented in this study is publicly available on Gene Expression

Omnibus56, under accession number GSE91378. Datasets for genomic DNAs are available at the European Nucleotide Archive accession number PRJEB22668 and scripts at linked sites. The authors have no competing interests. We thank Maria-Elena Mannarelli for technical support, Nick Barton for suggestions on the manuscript and Alexandra Rebocho for helpful discussions. This work was supported by BBSRC grant BB/G009325/1 awarded to E.C. Supplement contains additional data.

\section{Supplementary Materials}

Materials and Methods

Figs. S1 to S8

Table S1

References (30-58) 


\section{Fig. 1. Flower color pattern phenotypes}

Flower face (left) and side (right) views of A.majus (A.m.) species, showing lower ventral (V), lateral (L), and upper dorsal (D) lobes. Bee vision is sensitive to both yellow and the blue component of magenta reflectance. (A) A.m.pseudomajus. Magenta with yellow highlight. White arrows indicate bee entry point. (B) A.m.striatum. Yellow with magenta highlights. (C) Flowers from plants with ros EL from A.m.striatum (ros $\left.E L^{S}\right)$ and $S U L F$ from A.m.pseudomajus (SULF ${ }^{P}$ ). (D) Schematic showing the pathways to anthocyanin and aurone pigments; chalcone synthase (CHS), chalcone isomerase (CHI), A.m. chalcone 4'-O-glucosyltransferase (Am4'CGT) and A.m. aureusidin synthase (AmAS1).

\section{Fig. 2. SULF locus shows homology to Am4'CGT and signatures of selection}

(A) SULF inverted duplication. Organisation of Am4'CGT is shown twice (grey arrows) to indicate regions of homology with SULF (CDS = coding sequence). The left and right inverted repeats at SULF (red arrows) flank the transposon insertion site of sulf-660 (black triangle).

(B) Maximum likelihood phylogeny of CGT-related DNA sequences from Antirrhinum majus (red), Mimulus guttatus (black) and Linaria vulgaris (blue). Bootstrap support for

nodes with $>85 \%$ support (red circles, scaled by strength). For extended clade see fig. S2. (C) Plot of A.m.striatum sequence coverage normalized against A.m.pseudomajus for pools located at either end of the hybrid zone. Bars indicate genes, with SULF locus in red. Double-headed arrow shows region under-represented in A.m.striatum. Positions of KASP SNPs used for cline analysis (blue dots). 
(D) Clines for KASP markers across the hybrid zone transect. SNP index and chromosome position is indicated above each plot. Markers from SULF show steep clines at the hybrid zone, aligned with clines for ROS1 (right). Markers further away from SULF either show no clines (two examples shown), or clines centered at other geographic locations (fig. S4).

\section{Fig. 3. SULF locus makes small RNAs targeting Am4'CGT.}

(A) Comparison of total read abundance for small RNAs isolated from libraries of sulf660 and SULF-661. Small RNAs mapping to the SULF locus in red..

(B) Abundance of small RNAs mapping to SULF from the SULF-661 libraries. Reads with potential to target Am4'CGT (red) and those unable to target (too many mismatches) (grey).

(C) Blot of petal RNA probed with an oligo matching one of the abundant 21-mers, showing signal in ventral and lateral (VL) or dorsal (D) petals in SULF-661 but not sulf660. U6 = ubiquitin control.

(D) Complementary expression pattern of SULF small RNAs and Am4'CGT expression. Petals (left panel) were dissected into a central (C) yellow region, and peripheral (P) nonyellow region. For SULF expression, small RNA blots were probed with SULF, revealing stronger expression in the peripheral compared to central region (middle panel). For Am4'CGT, RNA was subject to qRT-PCR showing lower expression in the peripheral region (right panel).

(E) Floral bud of A.majus was sectioned to reveal the pigments (top panel), and similar sections probed to show in situ expression of Am4'CGT (purple stain, bottom panel). 
(F) q-RT-PCR on petal RNA (total, or dissected into upper and lower regions). Expression of Am4'CGT is reduced in genotypes carrying SULF from A.majus (SULF ${ }^{M}$ ) or A.m.pseudomajus $\left(S U L F^{P}\right)$ compared to those carrying sulf from A.majus (sulf $f^{\mathfrak{Y}}$ ) or A.m.striatum (sulf $f^{\S}$ ). Standard errors calculated from the means of 3 independent biological samples, each analyzed in triplicate.

\section{Fig. 4. Expression and frequency distribution of inverted repeats and microRNA genes in Antirrhinum majus}

(A) Frequency and expression levels of inverted repeats with folding energies similar to SULF, as a function of length of predicted hairpin RNA (including spacer). An inverted repeat is considered expressed if the maximum overall abundance of incident sRNAs, in any library is above a noise threshold (20). Boxed region shows class to which SULF belongs.

(B) Average complexity and mean length of small RNAs mapping to inverted repeats (as in A) and microRNA hairpins. Each point corresponds to a predicted transcript with a hairpin-like structure. SULF hairpin circled in red. Only sRNAs in the 21-24nt range are considered. Average complexity is the number of different reads (non-redundant) divided by the total number of reads mapping to the hairpin (29). Although SULF generates small RNAs throughout the inverted repeats, the high abundance of some leads to a low overall complexity. For inverted repeats, transcript abundance is color coded on a log scale and varies from blue (low abundance, 20) to red (high abundance,160,000). Orange indicates microRNA hairpins. 


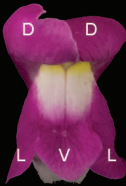
ROS $^{p} \mathrm{I}^{p}$ SULF $^{p}$

B

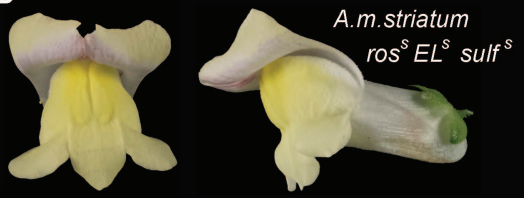

C
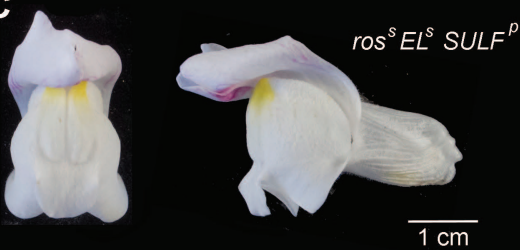

D

1 p-coumaryl-CoA + 3 malonyl-CoA
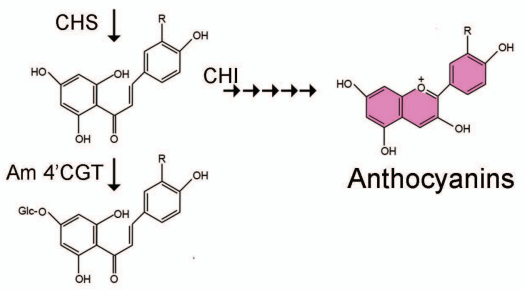

Anthocyanins

Am AS1 $\downarrow$

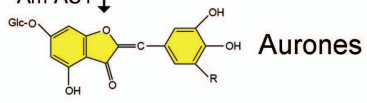


A

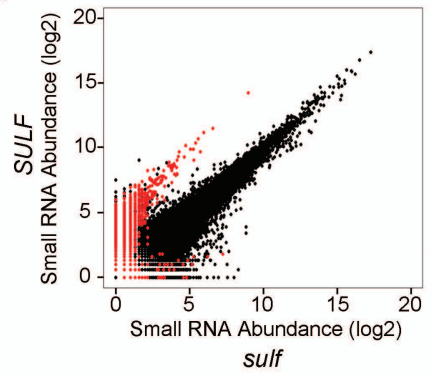

$\frac{\text { sulf }}{\mathrm{VL} \quad \mathrm{D}} \frac{\text { SULF }}{\mathrm{VL} \quad \mathrm{D}}$

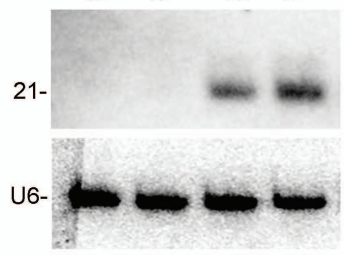

E

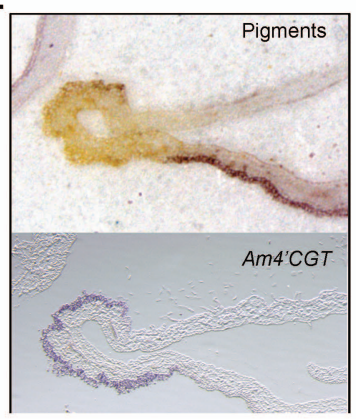

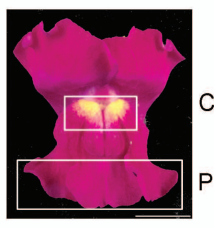

D

B

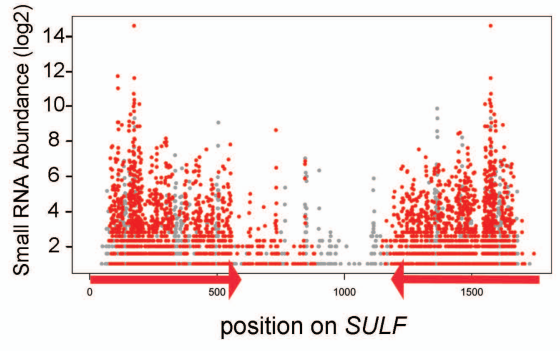

Am4'CGT mRNA

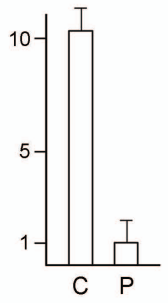

F

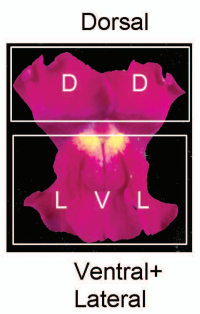

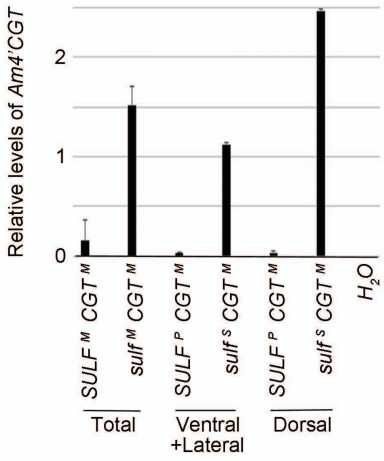




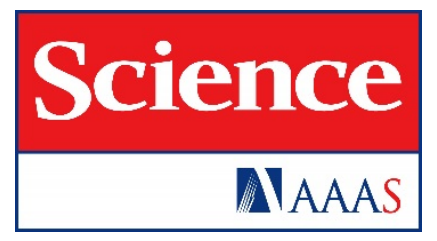

\section{Supplementary Materials for}

\section{Evolution of flower color pattern through selection on regulatory small RNAs}

Desmond Bradley, Ping Xu, Irina-Ioana Mohorianu, Annabel Whibley, David Field, Hugo Tavares, Matthew Couchman, Lucy Copsey, Rosemary Carpenter, Miaomiao Li, Qun Li, Yongbiao Xue, Tamas Dalmay*, Enrico Coen*

*Corresponding authors. Email: enrico.coen@jic.ac.uk ; t.dalmay@uea.ac.uk

\section{This PDF file includes:}

Materials and Methods

Figs. S1 to S8

Tables S1 


\section{Materials and Methods}

\section{$\underline{\text { Plant Material }}$}

Populations flanking a hybrid zone in the Pyrenees (2) provided seed for maintaining lines of A.m.pseudomajus $\operatorname{ROS}^{P} S U L F^{P}$ (from Ventola) and A.m.striatum $\operatorname{ros}^{S} \operatorname{sulf}^{S}$ (from La Molina). Greenhouse plants were grown as described (1), with supplemental lights in winter to give 12-16h days or grown outside in summer upon benches.

Antirrhinum majus stock lines are highly inbred and maintained at the John Innes (JI) Centre. Stocks JI7 and JI75 are INC SULF, while JI57 is inc sulf. The inc mutation reduces magenta (anthocyanin) pigments in the flower(2), while sulf results in spread yellow through the petal lobes $(3,4)$.

JI7 has been sequenced to give a chromosome-build reference genome (BGI, Beijing; http://bioinfo.sibs.ac.cn/am). JI75 has highly active endogenous transposons to generate mutants (5-7). JI660 sulf arose from a large-scale mutagenesis experiment using JI75, colorand JI661 SULF revertant arose from sulf-660 due to transposon instability.

\section{$\underline{\text { A.majus }} \underline{\text { crosses }} \underline{\text { to }} \underline{\text { species }}$}

A.majus JI7 was crossed with A.m.pseudomajus J1428 progeny; seed of J1428 was collected at 4,737.5m east of the HZ center (2). F1 plants were self-pollinated due to selfcompatible A.majus alleles to give family J108. KASP genotyping (see below) of the $\mathrm{J} 108$ family of 245 plants identified 15 plants with genotype $S U L F^{\mathrm{P}} A m 4{ }^{\prime}{ }^{\prime} C G T^{\mathrm{M}}$. A.majus JI7 was crossed with A.m. striatum J1160 progeny; seed of J1160 was collected at 12,364.2m west of the HZ center. F1 plants were self-pollinated to give family J106. KASP genotyping of the J106 family of 249 plants identified 23 plants with genotype sulf ${ }^{\mathrm{S}} \mathrm{Am} 4^{\prime} \mathrm{C} C \mathrm{~T}^{\mathrm{M}}$.

\section{Genotyping Crosses}

The KASP method (LGC Genomics) was used to identify SULF and Am4'CGT for introgression in F2 families J106 and J108 using the following oligo sets: Am4'CGT oligo set: do.238- GTTGAGATGCCCGGGTTCCCATTG (to detect P or S allele on Chr2:71834391-71834413); do.239-GTTGAGATGCCCGGGTTCCCATTA (to detect $M$ allele); do.240- ACTCATTGGTAAATCAGAGGAGTG (the common reverse oligo on Chr2:71834418-71834441).

SULF oligo set: do.256- CCACTCCGCGACCCATTGAGCT (to detect M allele on Chr4:34930351-34930372); do.257- CCACTCCGCGACCCATTGAGCC (to detect P or S allele); do.258- TCGATGGGATGATGATGTTAATGG (the common reverse oligo on Chr4:34930324-34930347).

KASP Genotyping was performed as described (LGC) and the fluorescence signals discriminating the two alleles was detected in a BioRad CFX96 lightcycler and data processed with the BioRad CFX Manager software v3.1.

Leaf genomic DNA Isolation.

3-6 small young leaves ( $\sim \mathrm{cm}$ long; total 100-200mg) were collected in eppendorf tubes and frozen in liquid nitrogen. Large numbers were collected in 96 tube format on 
dry ice or placed in glassine seed bags and dried in silica for long term storage at room temperature. Wild samples were collected in bags in silica gel.

Genomic DNA minipreps used the DNeasy Plant Mini Kit or DNeasy 96 Plant Kit according to instructions (Qiagen). For WGS, 2-5g of leaves were collected on dry ice and isolated by CTAB preps as described (8).

\section{Illumina resequencing}

Full details of the analysis pipelines and associated scripts are available here (https://github.com/JIC-CSB/antirrhinum-hz-pipeline). In short, paired end illumina reads were filtered and trimmed for quality using fastq-mcf (ea-utils v.1.1.2-484) or Trimmomatic(v0.32) and mapped to the JI7 reference genome (vIGBDV1) using Stampy (v.1.0.28) All default settings were used except the setting of an explicit substitution rate to account for expected divergence from the reference (--substitutionrate 0.02 in A. majus and A. majus subspecies pools, 0.05 in A. majus x A. sempervirens cross). Alignment file manipulations used SAMtools v1.3.0. After mapping, duplicate reads were excluded using the MarkDuplicates tool in Picard (v1.134; http://broadinstitute.github.io/picard) and local indel realignment using IndelRealigner was performed with GATK(v3.5.0).

\section{$\underline{\text { SULF }} \underline{\text { Isolation }}$}

\section{Mapping SULF}

We mapped the sulf locus using a sulf allele from A.sempervirens crossed to A.majus JI7. This population provided clear segregating phenotypes and a large number of useful SNPs for mapping. A.sempervirens Accession AC 1170 was backcrossed to A.majus JI7 three times before self-pollinating and generating a population of sulf/sulf and SULF/plants. Genomic DNA from pools of 35 sulf/sulf and 35 SULF/- individuals were resequenced and reads mapped to the reference genome. For analysis of homozygous SNP density, allele counts for reference (JI7) and alternate (A. sempervirens) alleles were exported using SAMtools mpileup with the following settings: -q 40 -Q 30 -BA -t AD. BCFtools (v1.3.1) was used to export a vcf of all variable sites, indels excluded, which was converted to table form using GATK VariantsToTable. Filtering thresholds were set following review of empirical distributions. We removed sites with depth $<10$ or $>100$ in either pool, sites that had a frequency of $<10 \%$ for the A. sempervirens allele in either pool and sites that were likely fixed homozygous for the alternate (A. sempervirens) allele. A $100 \mathrm{~kb}$ window analysis with a $50 \mathrm{~kb}$ step was applied genome-wide. The Homozygosity Index was calculated as the number of homozygous A. sempervirens sites (i.e. reference allele count equal to zero) divided by the total number of variable sites per window. Windows with fewer than 350 SNPs/100kb were excluded as these regions were deemed likely homozygous JI7. We identified a single genomic region with a high density of homozygous SNPs linked to yellow that mapped to $~ 3.2 \mathrm{Mb}$ chromosome 4 (fig. S1).

\section{Transposon-tagging SULF}

We used A.majus JI75, which has highly active endogenous transposons (5-7), to isolate a sulf mutant JI660 in a large-scale mutagenesis experiment. The sulf-660 phenotype had spread yellow, giving rise to an orange color through overlap with magenta (7) and crossing proved it was allelic to sulf JI57. The JI661 SULF revertant 
arose from sulf-660 due to transposon instability. Further independent SULF revertant plants were isolated; J216-3 and L142-11.

We used WGS to identify differences between sulf-660 and SULF-661 individuals which could be indicative of a transposon insertion specifically in the sulf genome using signatures from the distribution of reads with unmapped pairs. These criteria identified 187 loci which differed between sulf-660 and SULF-661 and which could reflect a transposon insertion in the sulf-660 line. Only one of these candidates, Chr4:3487992034879943, was also located in the region of high sequence differentiation between sulf/sulf and SULF/- pools.

To confirm that the sulf-660 line carries a transposon in this region we used long PCR (Phusion Taq as described by the manufacturer, New England Biolabs) with flanking oligos (do.99 and do.104) . do.99- TCTATCATGGCTTGATTTACAGCC (Chr4:34879576-34879599); do.104- TTTGCTTAGTGACTTTAACCACC (Chr4:34880053-34880075). The PCR products were cloned into pGEM-T (Promega) as described by manufacturers and Sanger sequenced. sulf-660 gave a 5kb product with homology to a CACTA transposon, consistent with insertion of a transposable element. These primers amplified a 500bp fragment in SULF-661, other independent SULF revertant individuals derived from sulf-660 and other $A$. majus stock lines. Three different excision footprints were found in the three independent revertants, all of which had restricted yellow phenotypes. The SULF region mapped to positions 34,877,442 34,880,992 on chromosome 4 (Fig. 2a), which lies within the 3.2Mb interval defined by genetic mapping described above.

\section{Genomic organization}

Annotated genes with homology to Am4'CGT were identified via BLASTp searches of the A. majus reference genome and the Mimulus guttatus reference v2.0 (via Phytozome v12.1) with an e-value threshold of $<1 \mathrm{e}^{-60}$. A single Linaria vulgaris 4'CGT accession (BAE48240.1) was also included (fig. S3). In addition to blastp hits to annotated proteins, regions of A.majus Chr4 with >500bp homology to Am4'CGT coding sequence were included in DNA alignments (Fig. 2B). Peptide alignments were generated using MUSCLE and DNA alignments using MAFFT both implemented using default settings via the EMBL-EBI multiple sequence alignment website (http://www.ebi.ac.uk/Tools/msa/). Substitution model evaluation and phylogeny construction with Maximum likelihood were performed using MEGA 6. In both cases, alignments were analyzed with partial deletion (site coverage cut-off 75\%) and support evaluated with 1000 bootstrap replicates. Resultant trees were visualised using iTOL (https://itol.embl.de/). Gene abbreviations used in Figure 2B are: AmC5 = AnMG048860500; MgC6 = Migut.F00274; MgC1 = Migut.F00273; MgC2 = Migut.H00709; MgC5 = Migut.F01071; MgC4 = Migut.F01069; MgC3 = Migut.F01068; AmC3 =AnMG0208611300; AmC2 = AnMG0308611900; AmC1 = AnMG0308612500. Nonannotated but homologous regions were Am H1 = Chr4:34868392-34867516; Am H2 = Chr4:34898959-34898266.

Deletion analysis 
Copy number across Chromosome 4 was investigated by comparing coverage depth in pooled sequence datasets from two pools located at either end of the hybrid zone (YP4 and MP11). These pools comprised 50 individuals each. Median coverage values were extracted using GATK (v3.5.0) depth of coverage for windows of 5kb with $1 \mathrm{~kb}$ step size for a $1 \mathrm{Mb}$ region of Chromosome 4 . The median coverage of each sample for the entire chromosome was used to correct for differences in sequencing effort for samples and the adjusted median coverage of YP4 was then divided by MP11, with results shown in Fig. 2c.

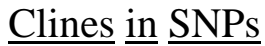

KASP genotyping platform (LGC Genomic) was used to genotype SNP loci adjacent to SULF (and other regions of chromosome 4) to quantify the steepness of clines in allele frequencies and their coincidence with flower color phenotype. We sampled 1722 plants over 3 years (2013, 2014 and 2015) from a natural hybrid zone between A.m.pseudomajus and A.m. striatum in the Spanish Pyrenees . Individuals were located to within 2 meters with a GPS (Trimble GeoXT datalogger), leaf tissue collected for DNA extraction and one flower taken for phenotyping (see below). To design SNP markers, potential divergent SNP loci were first identified from six WGS Illumina poolSeq analyses, each of 50-52 individuals. Pools YP4, YP2 and YP1 were harvested 12.8, 1.6 and $1.8 \mathrm{~km}$ west respectively, of the hybrid zone center (Latitude 42.32270, Longitude 2.07442). Pools MP11, MP4 and MP2 were from 8, 1.4 or 0.7 km east, respectively, of the center. SNPs were then selected on the basis of excess allele frequency differences in the outermost pools $\left(\Delta p_{1,6}>0.6\right)$, a minimum depth of 20 reads in all six pools for $100 \mathrm{bp}$ either side of the focal SNP. We used these criteria to design 35 KASP oligos (7 within 185kb of SULF and 28 across chromosome 4) with custom R and Python scripts (SNPextract.py (https://github.com/dfield007/snapdragon). This identified SNPs positions suitable for KASP genotyping platform (LGC Genomics) and extracted the 100bp sequence surrounding each candidate SNP. This included selecting sites with: (i) 30 $<$ depth $<300$ in both outer pools at the focal SNP (to reduce the probability of false positives and paralogs), (ii) $30<$ depth $<300$ for sequences 50bp either direction of focal SNP, (iii) $<3$ other SNPs within 50bp (to ensure primer efficiency), and (iv) biallelism (a KASPar requirement).. DNA extractions and genotyping were carried out by LGC Genomics. Replicate DNA extractions and genotyping of $n=500$ individuals confirmed relatively low error rates (mean $\sim 0.15 \%$ ). Plants were grouped into discrete demes of 200 x 200 meters, and their position collapsed along 25km one-dimensional transect. We fitted a five parameter, symmetric sigmoid cline to the observed genotype counts with the expected allele frequencies $\hat{p}$ along the one-dimensional transect as,

$$
\hat{p}=p_{0}+\frac{\left(p_{1}-p_{0}\right)}{1+\exp \left(-4\left(\frac{x-c}{w}\right)\right)}
$$

where $x=$ spatial coordinates in meters along the transect, $c=$ cline center, $w=$ cline width (1/gradient), $p_{0}=$ allele frequency at the asymptote in the west (A.m. striatum parental allele frequency) and $p_{1}=$ allele frequency at the asymptote in the east (A.m. pseudomajus parental allele frequency). In addition, we fitted a beta-binomial error term 
to account for the variance in allele frequencies across demes and to control for population structure along the cline $F_{S T}=\operatorname{var}(p) / \bar{p}(1-\bar{p})$.

To fit clines to the data, we used a metropolis-hastings (simulated annealing) algorithm to sample the likelihood surface following (9) with a few modifications. Briefly, we begin with a random set of parameters which are changed randomly and the $\log$ likelihood $\ln L$ is computed at each iteration. When the next iteration $\ln L^{\prime \prime}$ has a greater $\log$ likelihood than the previous likelihood $\ln L^{\prime}$ (i.e. $\ln L^{\prime \prime}>\ln L^{\prime}$ ), the new parameters are accepted. If the next iteration is lower $\left(\ln L^{\prime \prime}<\ln L^{\prime}\right)$, we accept with a probability $\ln L " / \ln L '$ '. To ensure ample exploration of the likelihood surface, the jump size for the next set of parameters are adjusted by a factor of 1.05 when accepted (accept scale) and by (1/1.05) when parameters are rejected (reject scale). After some tests of different accept and rejection scales, we found these values achieved efficient mixing and exploration of the likelihood surface with an acceptance rate $\sim 0.5$. This algorithm was run for 50,000 iterations with a burnin $=2000$. From this we found the best fitting combination of parameters from the maximum $\log$ likelihood $(\max \ln L)$. To find the 95\% credible regions $(95 \% \mathrm{CI})$ we assumed the likelihood surface follows a chi-square distribution (max $\ln L-2$ ). For each locus, we visually inspected the likelihood surface to ensure they were well mixed for each parameter. The algorithm was run on each locus three times with randomly chosen starting parameters to ensure independent runs displayed similar parameters estimates with overlapping 95\% CI.

center

$\underline{\text { PCR }} \underline{\text { Marker Genotyping }}$

A PCR genotyping assay distinguished A.m.pseudomajus and A.m.striatum flanking populations using the SULF PCR Marker do172-do156 that spanning the inverted repeats region: do.172- AAGTTCATCGCTCTTCAATCTCC (Chr4:34878913-34878935); do.156- TGAGGTGGCTAAATAGTGACCAC (Chr4:34881137-34881159) using standard PCR conditions with annealing at $55^{\circ} \mathrm{C}$ and extension of $2 \mathrm{~min}$. Control oligos to Am4'CGT were used to confirm gDNA presence; do.245- ATATCACCAACCACCCCATGC (Chr2:71833617-71833637) and do.259TGTTATACGTTTGCGACTCACGAGC (Chr2:71835432-71835456).

Populations in fig.S3 were A-D, A.m.striatum, at $\sim 13,11,7$ and $3 \mathrm{~km}$ west of the Hybrid Zone center, and E-G, A.m.pseudomajus, at 5, 8 and $8 \mathrm{~km}$ east of center.

\section{$\underline{\text { RNA analysis }}$}

We compared tissues from JI7 SULF, sulf-660, SULF-661, A.m.pseudomajus and A.m.striatum. Total RNA was isolated from late developmental stages of petals from young buds to just before opening (stages 3 to 6 of Davies et al.) (29). Each sample contained petals from 5-6 buds ( 200-300 mg tissue). Petals of 5-6 flower buds just before opening (stage 6) were cut at the tube lobes boundary, and separated into the upper dorsal lobes separate from the lower ventral/laterals. yellow foci) from the peripheral tissues (edge of lateral and ventral lobes) from 20-24 newly opened flowers.Total RNA was isolated from pooled tissue ( 100-200 mg) using RNeasy Plant mini Kit (Qiagen).

To purify small RNAs for library construction we first isolated total RNA.from petals sulf-660, SULF-661, A.m.pseudomajus or A.m. striatum. RNA was extracted using 
TriReagent (Ambion) following the manufacturer's protocol with minor modifications as follows. After Tri-reagent and chloroform extraction, the aqueous phase was mixed with an equal volume of isopropanol and stored at $-80^{\circ} \mathrm{C}$ overnight. The total precipitated RNA, was washed with $80 \%$ ethanol twice and re-suspended in RNase free $\mathrm{H}_{2} \mathrm{O}$ followed by a second round of phenol-chloroform extraction. The sRNA fraction was enriched using mirVana miRNA isolation kitTM (Ambion) according to the manufacturer's instructions.

One $\mu \mathrm{g}$ of the enriched sRNA fraction were used for sRNA library construction based on a previously published protocol (10). The libraries were sequenced on Hiseq2500 at Earlham Institute, Norwich, UK.

SULF-derived sRNA molecules were detected through RNA blot analysis as described (11). Five to eight microgram of total RNA were denatured and loaded onto $16 \%$ urea-polyacrylamide gels and the RNA was transferred to Hybond NX (Amersham) membrane through semi-dry electrophoresis. Chemical cross-linking used 1-ethyl-3 (dimethylaminopropyl) carbodiimide. The SULF inverted repeats (IR) were cloned in pGEM-T vector (Promega). The linearized and blunted templates were used to generate complementary RNA strands for both repeats. The RNA probes were labelled with $[\alpha-$ 32P] UTP using T7 or Sp6 RNA in vitro transcriptase (NEB) at $37^{\circ} \mathrm{C}$ for one hour. The reaction included: 1xRibomix, $1 \mathrm{mM} \mathrm{A}, \mathrm{C}, \mathrm{GTP}$ and $4 \mu \mathrm{M}$ UTP, 200ng of DNA template, 20units of RNA polymerase, and $0.2-1 \mu \mathrm{M}[\alpha-32 \mathrm{P}] \mathrm{UTP}$. To detect specific sRNAs, the complementary oligo DNA was end labelled with [ $\gamma$-P32] ATP using T4 DNA kinase (NEB).

To map cleavage sites of Am4'CGT mRNA in sulf-660 or SULF-661, 80 $\mu \mathrm{g}$ of total RNA was isolated from each and used for mRNA isolation with Dynabeads mRNA direct kit (Ambion) following the manufacturer's protocol. The cleavage sites of Am4'CGT mRNA were determined by 5'RACE using the Generacer kit (Invitrogen) as described (12). The Am4'CGT primary oligo used was SNAP-3-GSP 5'-

CTGTTGTGCTGAGAACGCTCCTCTTCTTCC (Chr2:71834715-71834744); followed by nested primer SNAP-3-targnest 5'-

CTCTTCTTCCGAAACAAAGGAAAATCACGCT (Chr2:71834694-71834724).

As above, total RNA was isolated from A.m.striatum and A.m.pseudomajus to determine any cleavage sites. The first oligo was GSP-0505 5'-

CCTCTGCTCTGCGTACAACGGCCAACC (Chr2:71834997-71835023); followed by nested primer GSP-9003 5'- GACTCAACACCTCTTTCTGCGGCACCC (Chr2:71834890-71834916).

\section{Expression analysis}

Based on the genomic sequence at the SULF locus, two primers near the inverted repeats were designed for RT-PCR analysis. About 250ng of mRNA isolated from ventral petals of SULF-661 and sulf-660 was used for reverse transcription reactions where polydT(20) was used as the reverse primer. Sequence specific primers: PX1 5'GTTCATCGCTCTTCAATCTCCATCATTTTCATC-3' (Chr4:34878905-34878947) and PX2 5'-GAACATACCCACTTACTTGGACGTCAGTG-3' (Chr4:34880836-34880864) were used for sequence specific amplification of cDNA expressed from this locus. The cDNA fragments specific to SULF-661 were gel extracted and cloned into pGEM-T vector (Promega) for sequence analysis. 
For RNA in situ of Am4'CGT, a $0.51 \mathrm{~kb}$ insert of Am4'CGT was generated with oligos do.203 5'-AACATACCCACTTACTTCGACG (Chr2:71834277-71834298) and do.204 5'-TCGACATCCACTCTTCTCCAACC (Chr2:71834764-71834786) and cloned into pGEM-T (Promega) as described by manufacturer. The clone pD569 was used to generate antisense RNA probes for in situ as described (13).

For qRT-PCR, RNA was isolated and DNase treated as described (Qiagen). First strand synthesis on 0.5 ug of each genotype with SuperScript III was performed as described (Invitrogen). qPCR Am4'CGT ${ }^{\mathrm{M}}$ oligos used were AW6 and AW8:

AW6 5'-TCGATTTCTTTGGTTGGCCC (Chr2:71834783-71834802) and AW8 5'ATTGATCCTCTGCTCTGCGT (Chr2:71835010-71835029). GAPDH reference gene oligos were: GAPDH_1762 5'-CACGAGACGAGCTTCACAAA (Chr4:1223737812237359) and GAPDH_1781 5'- CTGCCATTAAGGAGGAATCG (Chr4:1223800512238015; Chr4:12238141-12238150). PCR conditions were as described (14). These conditions gave linear amplification at high efficiency for each set of oligos. We used 3 experimental and 3 biological replicates and relative values (log2 scale) were all compared to the same sample of sulf-660 made in each analysis.

\section{$\underline{\text { RNAseq Bioinformatics }}$}

mRNA-seq analysis

50bp single-end libraries generated reads that were quality-filtered as detailed above and mapped to the $A$. majus reference genome using tophat v. 2.0.4. We calculated normalized expression values (RPKM) for transcripts using the cuffdiff tool from the cufflinks package. Statistical analyses were performed using the $R$ package and custom $R$ scripts (15).

\section{smallRNAs}

The sequencing fastq files were converted to fasta format and reads without Ns were retained for further analysis. The evaluation of quality scores was conducted as in the FastQC suite [https://www.bioinformatics.babraham.ac.uk/projects/fastqc/]. The 3' adapter was trimmed using perfect string matching on the first 7 nucleotides of the adapter (TGGAATT); the HD signatures, 4 assigned nucleotides at the 3' and 5' end of the insert $(10,16)$ were also trimmed. Next, the files were converted from redundant to non-redundant format $(17,18)$ and the results were summarized into redundant and nonredundant size class distributions.

In non-redundant format, the reads were mapped to the JI7 reference genome allowing 0,1 or 2 mis-matches and 0 gaps using PatMaN $(19,20)$. The reads were also mapped to plant mature miRNAs and miRNA hairpins, retrieved from miRbase, release 21 (21). The sequencing depth of the small RNA libraries was $\sim 25 \mathrm{M}$ reads, with $\sim 14 \mathrm{M}$ reads matching to the reference genome, full length, with no mis-matches or gaps allowed. The abundances of the reads were normalized using the reads per total method (22-24). The replicate to replicate differential expression was called on offset fold change, offset $=20$ (empirically determined) $(18,25)$ calculated on the proximal ends of maximal confidence intervals built on the available replicates. The differential expression on loci (regions on the genome) was conducted using a simplified form of CoLIde (26) 
applicable on 2 samples, with 2 replicates each. The sRNA analysis was conducted using custom-made Perl and R scripts. The presence plots were created in R, v 3.4.0. The secondary structures were obtained using RNAfold, part of the Vienna RNA package (27).

A scatter plot was generated to show comparative distribution of abundances between sulf-660 and SULF-661 (Fig.3A). Sequencing reads were obtained from two samples with two biological replicates for each genotype (as above). Control replicate vs replicate plots showed reads mapped along the diagonal indicating similarity. The reads were mapped fill length with no mis-matches or gaps allowed using PaTMaN (19). The abundances were normalized using the per total approach. Average expression between bio reps was calculated as $\log 2(($ replicate $1+$ replicate 2$) / 2+1)$. These were normalized and mapped to the genome using full lengths, with no mis-matches or gaps allowed. The small RNAs derived from the SULF locus were marked in red. Reads mapping to the SULF locus were plotted (Fig.3B). The small RNAs which may target Am4'CGT, were predicted in line with the Allen rules (maximum 4 mis-matches between the sRNA and the target, with no mis-matches on the 10th-11th positions) and plotted in red (15). Small RNAs not fitting this criteria for targetting are plotted in grey.

\section{Genome scan for inverted repeats}

The scan of the A.majus genome for inverted repeats similar to the SULF was first conducted using existing software [Emboss, palindrome application (28), detectIR (29) and Lirex (30)]. Given the spacer region separating the inverted repeats in SULF, its inverted repeat structure was not detected with existing software. We therefore developed a new method based on a palindrome search coupled with the prediction of secondary structures for the proposed inverted repeats that allowed for spacer regions. Briefly, the genome was scanned using windows of variable length (from 100nt to 1600nt, in increments of 50nt). Consecutive windows had a 50nt overlap. Each window was first scanned for the presence of perfect palindromic motifs (5nt), and regions with $70 \%$ of palindromic hits, were then folded using RNAfold and the adjusted minimum free energy calculated. The adjusted minimum free energy is the minimum (optimal) free energy resulting from the RNAfold prediction, normalized per 100nt. This adjustment is performed to ensure comparability between the stability of structures of different lengths (e.g. using the amfe, a haipin-like structure of 100nt can be compared to a structure of 1000nt). Without this adjustment, longer structures will always have lower mfes because of the higher number of AT and CG pairs which contribute to the lower mfe. Windows with adjusted minimum free energy less than -40 were scanned for the presence of one mature stem (windows with multiple stems were discarded). This minimum energy was chosen based on the properties of SULF. By comparison, conserved microRNAs have a free energy -20 and are therefore largely excluded from the search. Because of computational time limitations, searches with minimum free energies of -20 could only be performed for lengths up to 500nt.

The analysis was conducted on inverted repeats with spacers (for the latter, the window was split into 3 equal regions and the secondary structure was predicted on the concatenated sequence of the first and third fragments). In this search, SULF was returned as encoding a potential 1450nt hairpin, which excluded the first $100 \mathrm{nt}$ of the 
region of contiguous 590 inverted homology. This exclusion arose because this region folded upon itself and such foldback regions were trimmed in the analysis.

The distribution of abundances of sRNAs (from the samples described above) incident with these inverted repeats is shown in Fig. 4a. Inverted repeats with an overall/total sRNA abundance less than 20 were considered to be expressed below the noise range, and thus classified as non-expressed. Only SULF showed strong differential expression between sulf-660 and its revertant. The microRNA hairpins used in Fig. 4b were identified by screening the small RNA libraries for matches to conserved plant microRNAs and then mapping them to the genome. The miRNA hairpins were determined using a similar approach as described previously (31). 
A

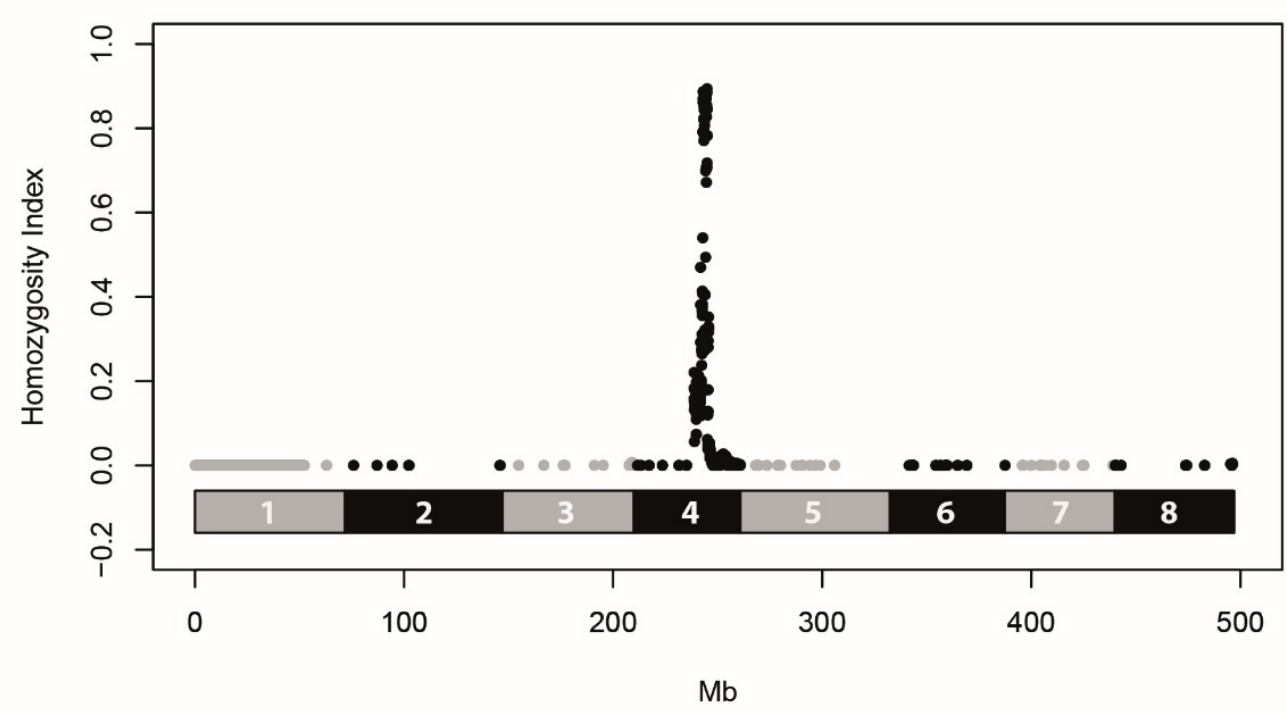

B

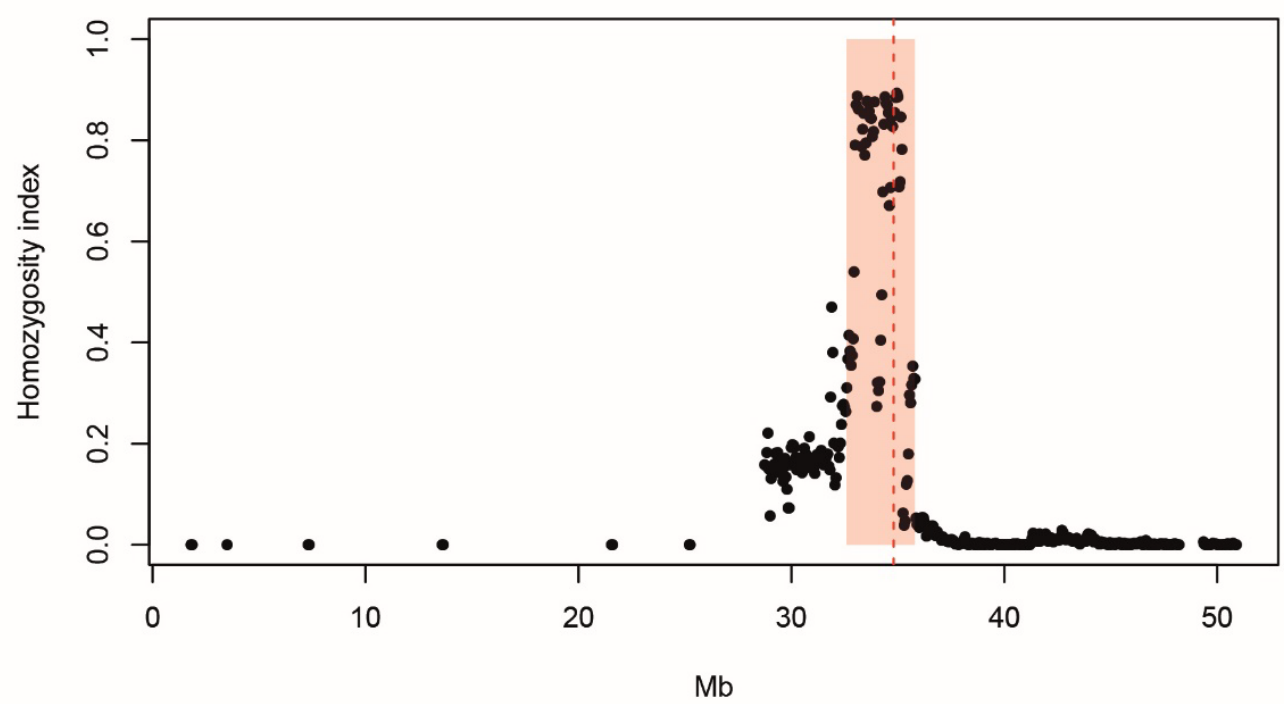

Fig. S1. SNP homozygosity density plot

(A) A genome-wide scan revealed a single major peak of local homozygosity on chromosome 4 . $100 \mathrm{~kb}$ sliding window analysis (50kb step size) of A.sempervirensderived homozygous SNP density in a pool of 35 individuals with the sulf phenotype selected from a segregating population. Regions with no datapoints are likely fixed for either JI7 or A. sempervirens alleles. (B) The chromosome 4 signal block was 3.2Mb wide (pink shading) and contained the SULF locus (dashed red line). 
A

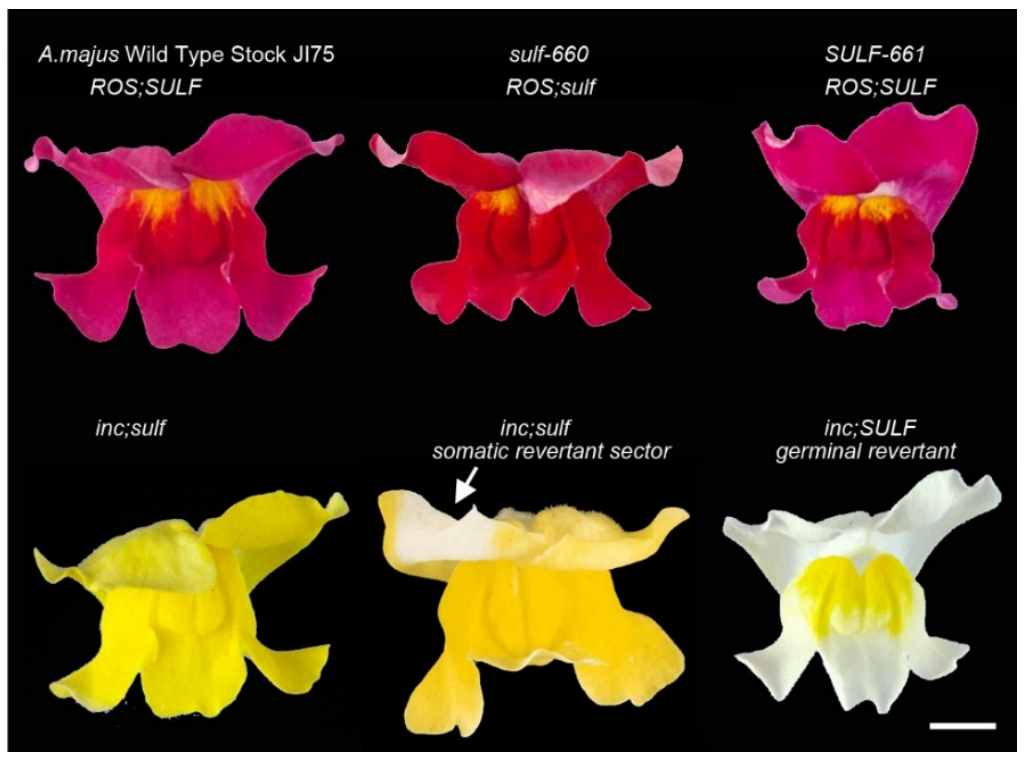

B

\begin{tabular}{|c|c|}
\hline & 4879930 \\
\hline & I \\
\hline Reference Genome A majus JI7 & ...GTATGC - . - AGAGC... \\
\hline Revertant 1 & ...GTATGC - -GCAGAGC... \\
\hline Revertant 2 & ...GTATGCATG -AGAGC... \\
\hline Revertant 3 & ...GTATGCATGCAGAGC... \\
\hline
\end{tabular}

Fig. S2. Isolation of the unstable sulf-660 allele in A.majus.

(A) Mutagenesis of the magenta stock A.majus JI75 ROS SULF line produced the orangered sulf-660 mutant. Instability gave rise to the revertant SULF-661, again magenta. To allow the change in yellow pattern to be more easily seen, sulf-660 was crossed with an inc mutant (which blocks magenta pigmentation) and an inc sulf plant isolated. Growing this plant and its progeny revealed instability in the sulf phenotype, both somatic and germinal, which was due to transposon excision from the sulf-660 locus. Scale bar $1 \mathrm{~cm}$. (B) Revertant SULF plants derived from the unstable sulf-660 were anlysed by PCR at the site of the transposon. Three revertants analyzed all showed loss of the transposon at the same site on Chromosome 4. and had three different sequence alterations (footprints) confirming that this locus is SULF. Note SULF-661 has revertant 2 sequence. 


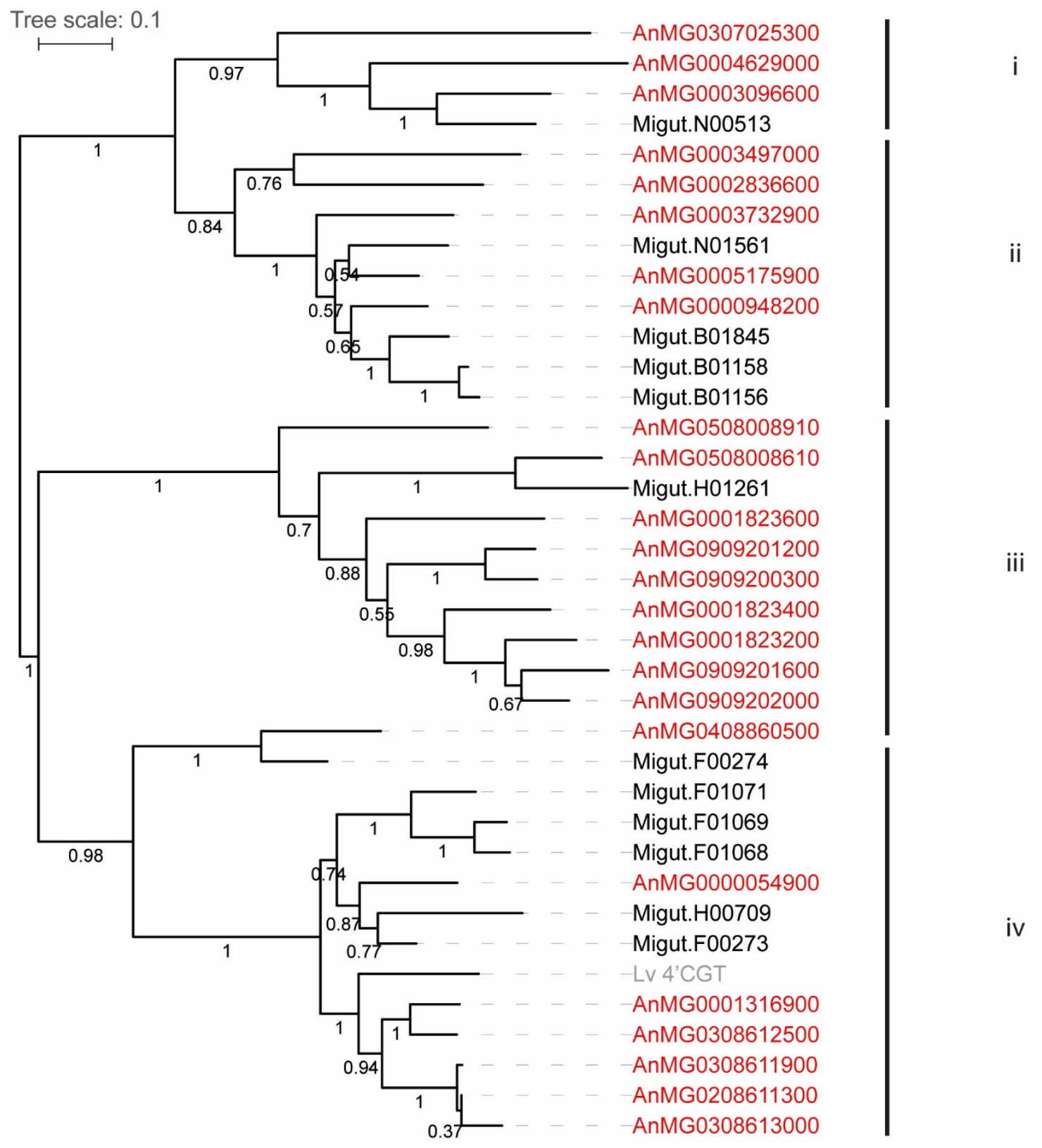

Fig. S3. Extended peptide phylogeny for CGT-like genes.

We compared CGT-likegenes from Antirrhinum (red), Linaria (grey) and Mimulus (black). AnMG0001316900 corresponds to Am4'CGT. Clade iv is plotted in Fig 2B Details given in Methods 


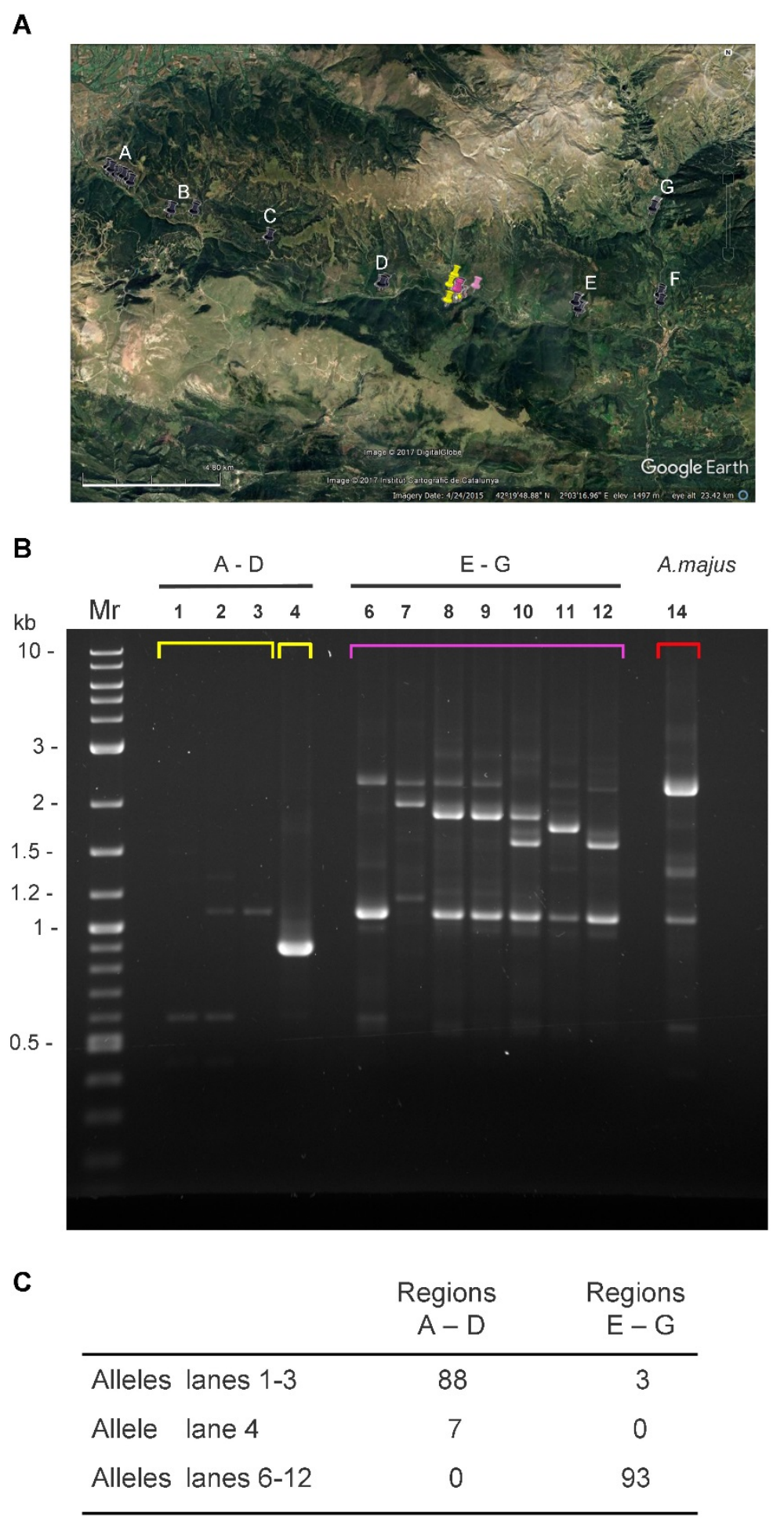


Fig. S4. A PCR marker identifies A.m.pseudomajus and A.m.striatum SULF alleles forming a sharp cline at a Hybrid Zone.

(A) 95-96 indivduals were analyzed from regions flanking a hybrid zone (central colored markers); regions A-D (A.m.striatum flank) and E-G (A.m.pseudomajus flank). (B) Individual gDNAs were compared to A.majus JI7 using the PCR Marker do172-do156 flanking the inverted repeats of the SULF locus. A range of PCR allele patterns were found, falling into 3 classes. The first allele class includes lanes 1-3 where no strong bands were found. The second allele class had only 1 major band, lane 4, and represents a deletion of $1.3 \mathrm{~kb}$ of the inverted repeats region. The last allele class was only seen for the A.m.pseudomajus flank and has a range of higher kb bands (lanes 6-14) similar to A.majus (lane 14). Size markers are given on left-hand side in kilobases (kb). (C) Frequencies of the 3 allele classes for each flank of the hybrid zone, regions A-D (west of center) and regions E-G (east of center). 
A
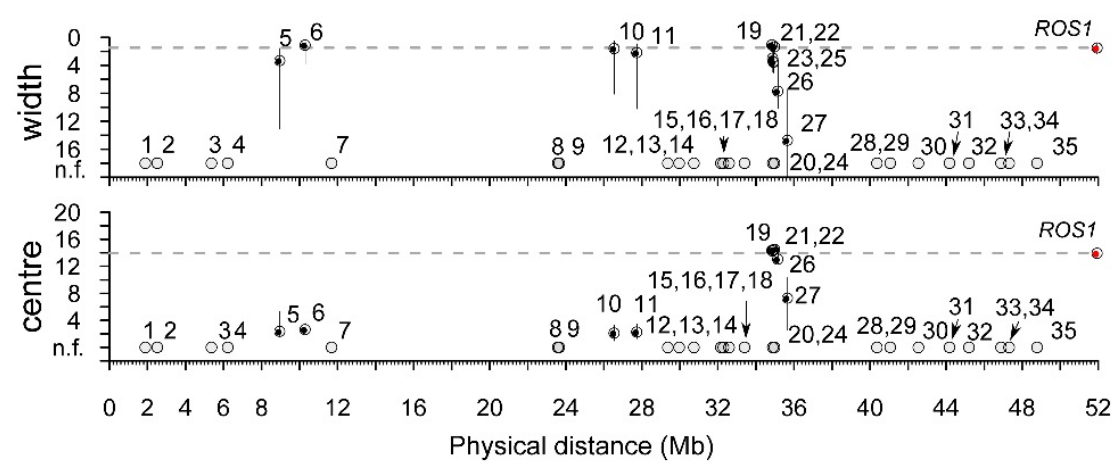

B
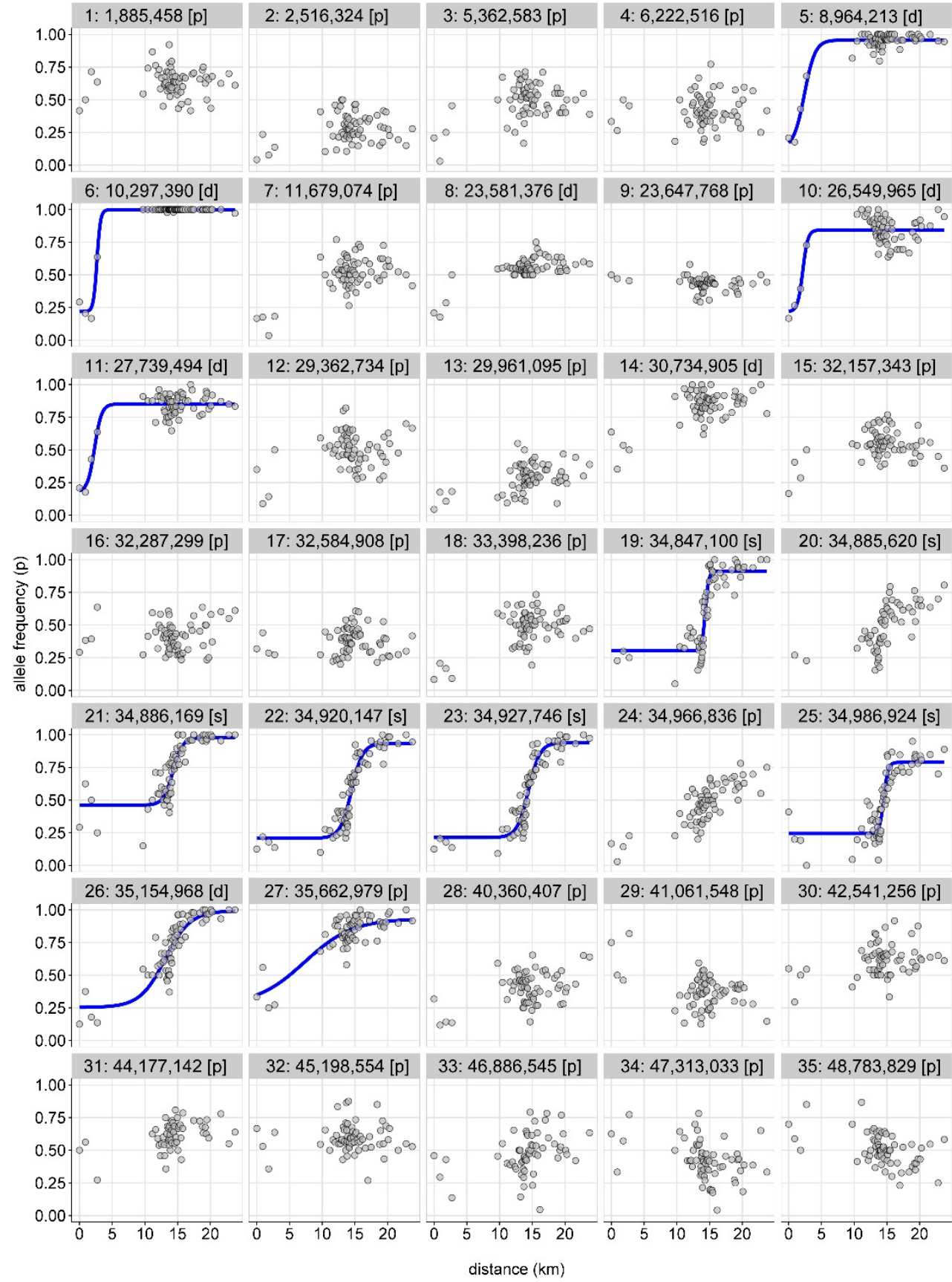
Fig. S5. Geographic cline fits for 35 SNP loci across Chromosome 4.

(A) Cline width and center (with 95\% confidence intervals) where filled circles indicate SNP loci with a cline fit $\left(\Delta p_{1,6}>0.6\right)$ and open circles indicate SNP loci where a cline fit was not performed due to low allele frequency differences $\left(\Delta p_{1,6}<0.6\right)$. SNP loci with no cline fits are also indicated with respect to their position along the y-axis (n.f. = no fit). Values for a ROS SNP, highlighted with a dotted line, shown for comparison. SNPs 1926 derive from the SULF region. (B) Individual SNP loci, with allele frequencies in 200 $\mathrm{m}$ demes in relation to distance along the transect. Best fitting cline model indicated with blue curve. SNP loci sorted in order across the chromosome from top left to bottom right. 
A
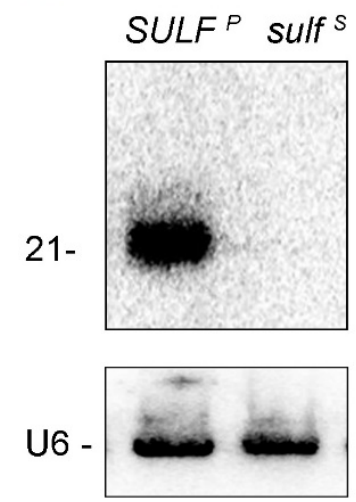

B
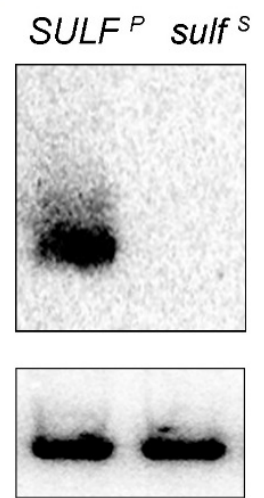

Fig. S6. Am4'CGT mRNA cleavage products in $S U L F$ petals.

Small RNA Blots for A.m.pseudomajus and A.m.striatum. Small RNAs were isolated from (A) A.m.pseudomajus (SULF ${ }^{P}$ ) and A.m.striatum (sulf ${ }^{S}$ ) species petals, or from (B) A.m.pseudomajus $\left(S U L F^{P}\right)$ and A.m.striatum (sulf ${ }^{\Im}$ ) introgressed into A.majus carrying Am4'CGT ${ }^{M}$. Blots were probed for SULF small RNAs and found 21 nucleotides (nt). Samples are from dorsal petals of 3-5 pooled independent plants. The same results were found for 2 further independent biological replicates in each case, or similarly from ventral petals. Blots also probed with Ubiquitinn U6 as a loading control. 


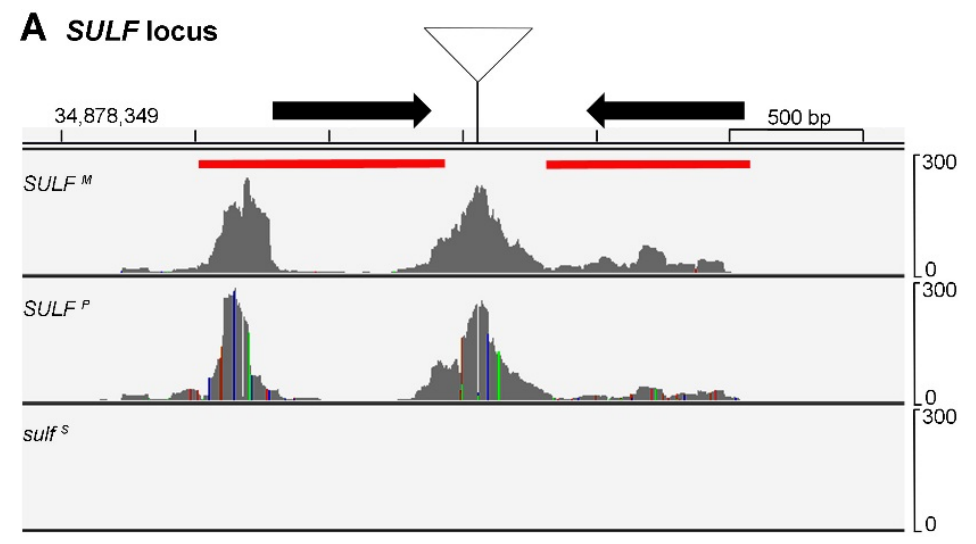

\section{B NIVlocus}

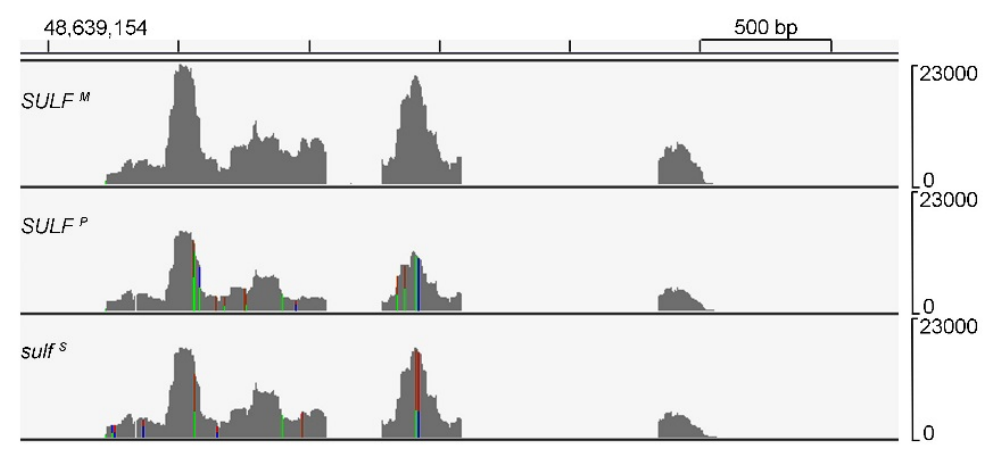

C

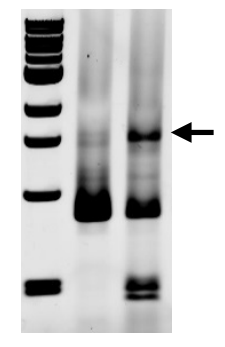

\section{Fig. S7. RNA mapping to the SULF locus}

(A, B) IgV profiles showing depths of mapped RNA reads at a, the SULF locus and b, the NIV locus as a control. RNAseq was made on total petals from small $10 \mathrm{~mm}$ long flower buds for A.majus JI7 SULF ${ }^{M}$, A.m.pseudomajus SULF ${ }^{P}$ and A.m.striatum sulf individuals. Reads are shown as grey peaks (with species SNPs colored). Depths are shown on the right-hand side. Both loci are on Chromosome 4 at the positions indicated. The inverted repeats at SULF are shown as large black arrows, with the site of the transposon insertion in the sulf-660 allele indicated by a triange (not to scale). (C) RTPCR identifed a $1695 \mathrm{nt}$ transcript (arrow) in petals of SULF-661 (lane 2) but not in sulf- 
660 (lane 1). Sequencing showed that this transcript mapped to the SULF above (red line in A). The transcript includes the inverted repeats, but splicing has removed part of the intervening region. Smaller transcripts were found that included only the first or seond repeat. The sulf mutant showed only transcripts containing the first or second repeat alone, but with some transposon sequence. 
A

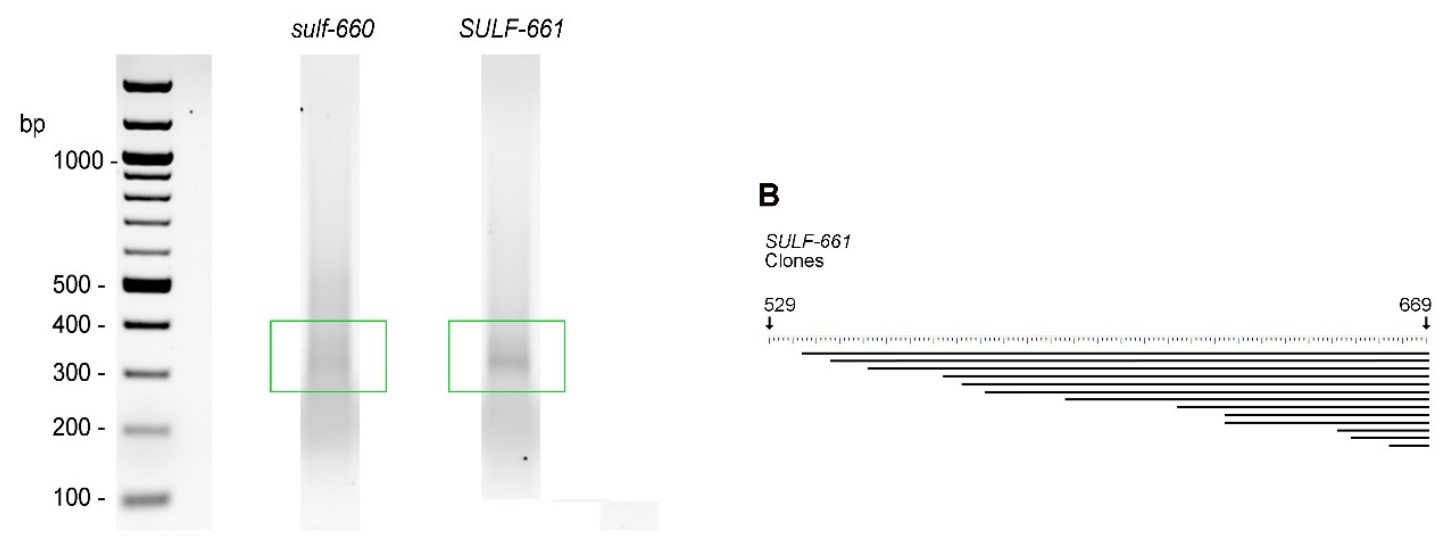

C

A.m.striatum A.m.pseudomajus

sulf ${ }^{s}$

SULF

=

sulf

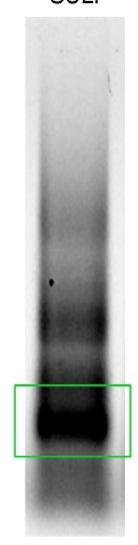

D

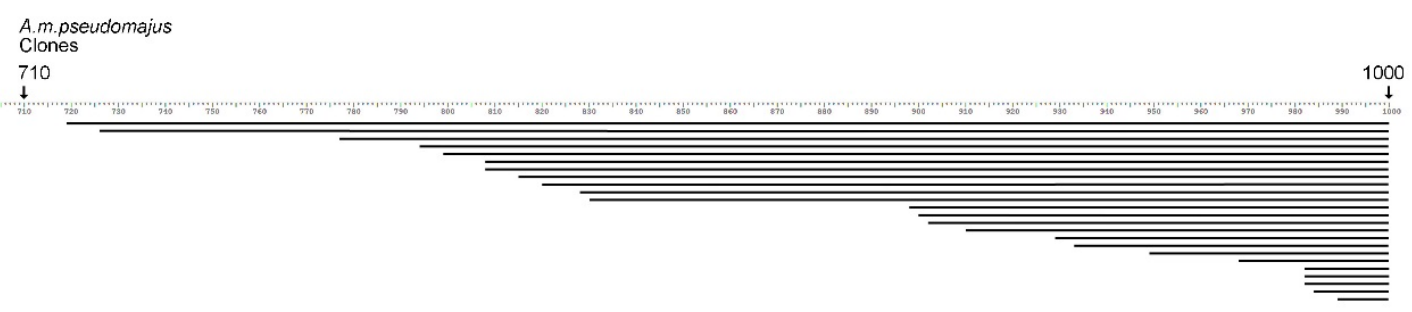

Fig. S8. Am4'CGT mRNA cleavage products in SULF petals.

5'RACE on total RNA from sulf and SULF petals. (A) Gel regions were isolated (green boxes), cloned and sequenced. sulf-660 gave few colonies and 3 clones sequenced did not contain Am4'CGT fragments. (B) SULF-661 gave many clones, 14 were sequenced; 1 had no insert, while 13 had fragments of $A m 4$ 'CGT that showed 12 different start site sequences (black bars), indicating a range of Am4'CGT mRNA fragments in SULF petals. Numbers are nucleotides relative to start number 1 of ATG. All start sites on left are as found. All sequences stopped at the same position (at the end oligo) but only sequence to nt 669 is shown. 
(C) Gel of 5'RACE products for A.m.striatum and A.m.pseudomajus. The clear band in A.m.psuedomajus (green box) was isolated and 35 clones sequenced; 27 had fragments of Am4'CGT, with 24 unique start sites shown. 


\begin{tabular}{|c|c|c|c|c|c|c|c|c|}
\hline $\begin{array}{l}\text { Reference genome } \\
\text { match }\end{array}$ & $\begin{array}{l}\text { Query } \\
(A m \\
4 \text { 'CGT) } \\
\text { match }\end{array}$ & $\begin{array}{l}\text { Percent } \\
\text { identity }\end{array}$ & $\begin{array}{l}\text { Aligned } \\
\text { length }\end{array}$ & $\begin{array}{l}\text { Mismatch } \\
\text { Count }\end{array}$ & $\begin{array}{l}\text { Gap } \\
\text { Coun } \\
t\end{array}$ & e-value & $\begin{array}{l}\text { Match } \\
\text { orientati } \\
\text { on }\end{array}$ & $\begin{array}{l}\text { Query region } \\
\text { overlap }\end{array}$ \\
\hline $\begin{array}{l}\text { Chr4:27714674- } \\
27714521\end{array}$ & $2706-2857$ & 72.78 & 158 & 33 & 4 & $2.00 \mathrm{E}-14$ & minus & downstream \\
\hline $\begin{array}{l}\text { Chr4:30856882- } \\
30856986\end{array}$ & 2042-2143 & 74.53 & 106 & 22 & 2 & 2.00E-09 & plus & CDS \\
\hline $\begin{array}{l}\text { Chr4:34781696- } \\
34783036 \\
\end{array}$ & $1017-2352$ & 74.43 & 1369 & 289 & 9 & 0 & plus & CDS \\
\hline $\begin{array}{l}\text { Chr4:34832879- } \\
34833033\end{array}$ & $608-755$ & 77.07 & 157 & 25 & 3 & $3.00 \mathrm{E}-25$ & plus & upstream \\
\hline $\begin{array}{l}\text { Chr4:34833584- } \\
34833806\end{array}$ & 134-365 & 72.5 & 240 & 41 & 11 & $2.00 \mathrm{E}-22$ & plus & upstream \\
\hline $\begin{array}{l}\text { Chr4:34834885- } \\
34834994\end{array}$ & 653-753 & 79.28 & 111 & 12 & 4 & $1.00 \mathrm{E}-16$ & plus & upstream \\
\hline $\begin{array}{l}\text { Chr4:34836385- } \\
34838049\end{array}$ & $789-2383$ & 81.58 & 1683 & 204 & 21 & 0 & plus & $\begin{array}{l}\text { promoter, } \\
\text { CDS, } \\
\text { downstream }\end{array}$ \\
\hline $\begin{array}{l}\text { Chr4:34845928- } \\
34846236\end{array}$ & 2415-2735 & 73.93 & 326 & 63 & 6 & $4.00 \mathrm{E}-49$ & plus & downstream \\
\hline $\begin{array}{l}\text { Chr4:34856749- } \\
34857066\end{array}$ & 533-815 & 64.63 & 328 & 61 & 11 & $1.00 \mathrm{E}-10$ & plus & upstream \\
\hline $\begin{array}{l}\text { Chr4:34861039- } \\
34860898\end{array}$ & 2153-2291 & 80.28 & 142 & 25 & 1 & $2.00 \mathrm{E}-28$ & minus & CDS \\
\hline $\begin{array}{l}\text { Chr4:34868392- } \\
34867516\end{array}$ & $1278-2165$ & 86.26 & 888 & 111 & 3 & 0 & minus & CDS \\
\hline $\begin{array}{l}\text { Chr4:34873993- } \\
34873478 \\
\end{array}$ & $789-1285$ & 81.24 & 533 & 47 & 11 & $\begin{array}{l}5.00 \mathrm{E}- \\
143 \\
\end{array}$ & minus & upstream, CDS \\
\hline $\begin{array}{l}\text { Chr4:34875836- } \\
34875294\end{array}$ & 128-682 & 73.37 & 597 & 63 & 18 & 1.00E-99 & minus & upstream \\
\hline $\begin{array}{l}\text { Chr4:34877442- } \\
34877628\end{array}$ & 171-383 & 74.07 & 216 & 24 & 6 & $9.00 \mathrm{E}-32$ & plus & upstream \\
\hline $\begin{array}{l}\text { Chr4:34878446- } \\
34878726\end{array}$ & $620-888$ & 72.11 & 294 & 44 & 13 & $1.00 \mathrm{E}-30$ & plus & upstream \\
\hline $\begin{array}{l}\text { Chr4:34878794- } \\
34878849\end{array}$ & $904-960$ & 88.14 & 59 & 2 & 3 & 2.00E-09 & plus & upstream \\
\hline $\begin{array}{l}\text { Chr4:34878900- } \\
34880045\end{array}$ & $1069-2235$ & 84.15 & 1186 & 129 & 12 & 0 & plus & CDS \\
\hline $\begin{array}{l}\text { Chr4:34880273- } \\
34880045\end{array}$ & $2585-2811$ & 78.39 & 236 & 35 & 5 & $2.00 \mathrm{E}-46$ & minus & downstream \\
\hline $\begin{array}{l}\text { Chr4:34880865- } \\
34880320 \\
\end{array}$ & 1389-1929 & 88.46 & 546 & 58 & 2 & 0 & minus & CDS \\
\hline $\begin{array}{l}\text { Chr4:34880992- } \\
34880907\end{array}$ & 882-973 & 86.96 & 92 & 6 & 3 & 7.00E-21 & minus & upstream \\
\hline $\begin{array}{l}\text { Chr4:34884145- } \\
34884056\end{array}$ & 1778-1868 & 87.91 & 91 & 10 & 1 & $5.00 \mathrm{E}-23$ & minus & CDS \\
\hline $\begin{array}{l}\text { Chr4:34885632- } \\
34885387\end{array}$ & $1443-1691$ & 83.13 & 249 & 39 & 2 & $6.00 \mathrm{E}-66$ & minus & CDS \\
\hline $\begin{array}{l}\text { Chr4:34887433- } \\
34887098\end{array}$ & $1093-1456$ & 71.47 & 368 & 69 & 10 & 4.00E-43 & minus & CDS \\
\hline $\begin{array}{l}\text { Chr4:34890973- } \\
34889921\end{array}$ & $155-1131$ & 70.77 & 1098 & 155 & 35 & $\begin{array}{l}6.00 \mathrm{E}- \\
148\end{array}$ & minus & upstream, CDS \\
\hline $\begin{array}{l}\text { Chr4:34898959- } \\
34898266\end{array}$ & $1862-2543$ & 80.51 & 708 & 98 & 14 & $\begin{array}{l}7.00 \mathrm{E}- \\
179 \\
\end{array}$ & minus & $\begin{array}{l}\text { CDS, } \\
\text { downstream }\end{array}$ \\
\hline $\begin{array}{l}\text { Chr4:34901729- } \\
34901618\end{array}$ & 2358-2462 & 76.79 & 112 & 19 & 3 & $3.00 \mathrm{E}-13$ & minus & $\begin{array}{l}\text { CDS, } \\
\text { downstream }\end{array}$ \\
\hline $\begin{array}{l}\text { Chr4:34917689- } \\
34917086\end{array}$ & $1297-1924$ & 74.8 & 639 & 115 & 6 & $\begin{array}{l}2.00 \mathrm{E}- \\
122\end{array}$ & minus & CDS \\
\hline $\begin{array}{l}\text { Chr4:34919018- } \\
34918765\end{array}$ & $1017-1294$ & 71.99 & 282 & 47 & 6 & 4.00E-36 & minus & CDS \\
\hline $\begin{array}{l}\text { Chr4:34921202- } \\
34920975\end{array}$ & $167-415$ & 68.5 & 254 & 49 & 11 & $9.00 \mathrm{E}-13$ & minus & upstream \\
\hline $\begin{array}{l}\text { Chr4:35191842- } \\
35193179\end{array}$ & $1017-2352$ & 74.74 & 1366 & 287 & 9 & 0 & plus & CDS \\
\hline
\end{tabular}




\section{Table S1.}

Homology between Antirrhinum majus Chromosome 4 and Am4'CGT (AnMG0001316900) and flanking regions as revealed by Blastn. Results were filtered to exclude alignments under 50bp in length or with an e-value of greater than $1.0 \mathrm{e}^{-9}$. The query sequence comprised the Am4'CGT single exon coding sequence (positions 10012374 with upstream and downstream flanks (positions 1-1000 and 2375-2874, respectively). Content shows defined loci as Fig.2c, with a (AnMG0307025300.01), b (AnMG0308611900.01), c (AnMG0308612500.01), d (AnMG0308613000.01), e (AnMG0308613000.01) and f (AnMG0208611300.01). 